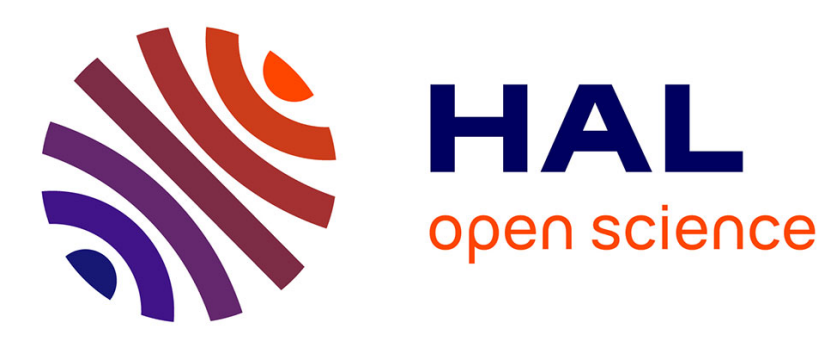

\title{
Logiques modales et anthropologie
}

Laurent Fontaine

\section{To cite this version:}

Laurent Fontaine. Logiques modales et anthropologie: Des règles à la parole chez les Indiens yucuna d'Amazonie colombienne. L'Homme - Revue française d'anthropologie, 2007, 184, pp.131-154. halshs00590728

\section{HAL Id: halshs-00590728 \\ https://shs.hal.science/halshs-00590728}

Submitted on 4 May 2011

HAL is a multi-disciplinary open access archive for the deposit and dissemination of scientific research documents, whether they are published or not. The documents may come from teaching and research institutions in France or abroad, or from public or private research centers.
L'archive ouverte pluridisciplinaire HAL, est destinée au dépôt et à la diffusion de documents scientifiques de niveau recherche, publiés ou non, émanant des établissements d'enseignement et de recherche français ou étrangers, des laboratoires publics ou privés. 


\title{
Logiques modales et anthropologie : \\ Des règles à la parole chez les Indiens yucuna d'Amazonie colombienne
}

\author{
Laurent FONTAINE \\ Membre du Laboratoire des langues et civilisations à tradition orale \\ (Lacito, UMR $7107 \mathrm{du}$ CNRS)
}

Confrontée aux phénomènes sociaux, toute recherche en sciences sociales ou toute intervention sur le terrain est nécessairement demandeuse de schèmes clairs et bien balisés pour les expliquer ou les anticiper. Ce qui devient aujourd'hui de plus en plus urgent à mesure que les politiques nationales et internationales exigent de la part des chercheurs une mise en relief de «l'utilité » de leurs investigations. Or, pour ne parler ici que du cas français, s'il existe un certain décalage entre cette pression des décideurs et les recherches en sciences sociales, c'est précisément parce que ces dernières ne se montrent pas assez «fonctionnalistes», et donc «fonctionnelles», non seulement vis-à-vis des gouvernements, mais aussi d'une large proportion de l'opinion publique.

Sans aborder davantage cet épineux problème institutionnel, nous le mentionnons uniquement pour souligner l'importance des méthodes qui permettent aux sciences sociales d'expliquer relativement simplement les fonctionnements des phénomènes sociaux, que l'on se place au niveau macro ou micro-sociologique.

Bien entendu, beaucoup de travaux progressent dans ce sens, mais il reste à éclaircir certaines articulations que les méthodes actuelles parviennent rarement à discerner : celles qui articulent fonctionnellement (par l'analyse de leurs effets) les idées et les pratiques, les conceptions et les organisations sociales, les systèmes culturels et les systèmes sociaux.

Pour expliquer de quelles manières les conceptions symboliques influencent les actions, il est crucial de ne pas seulement traduire les états ou les faits qu'elles représentent, mais de dégager les règles qu'elles emploient ou signalent plus ou moins implicitement. Une telle démarche rappelle bien sûr celle que préconisait Radcliffe-Brown pour «décrire la forme d'une structure sociale par les modèles de comportement auxquels se conforment les individus et les groupes dans leurs rapports avec autrui. » (1968, pp. 301302). Certes, sa méthode resterait critiquable si elle recommandait uniquement le recours à l'observation et la description des «relations sociales », car comme l'a parfaitement souligné Lévi-Strauss, les structures ne se déduisent pas des observations. ${ }^{1}$ Mais RadcliffeBrown ne s'est pas contenté d'énoncer cette unique règle de méthode, il considérait celle-ci indispensable pour comprendre «ces modèles partiellement formulés en règles d'étiquette, de morale et de droit, n'existant naturellement que lorsque les membres de la société les reconnaissent soit verbalement en les posant comme règle, soit en les observant directement. ». Selon lui, «le problème n'est pas l'existence de ces règles dans chaque société, mais leur fonctionnement dont la connaissance est nécessaire pour comprendre scientifiquement la société. » (Ibidem, p. 302).

Si l'on veut apporter quelques éléments de réponse à cette discussion, il est crucial de définir précisément le terme de règle. En priorité, il convient de remarquer que contrairement à un état ou un acte, une règle est nécessairement irréelle. En d'autre terme, elle n'a pas d'existence propre, elle ne peut apparaître qu'en tant que référence, c'est-à-dire

\footnotetext{
${ }^{1}$ Comme il le dit lui-même : « le principe fondamental est que la notion de structure ne se rapporte pas à la réalité empirique, mais aux modèles construits d'après celle-ci. » (Lévi-Strauss 1974, p. 331).
} 
uniquement par l'intermédiaire d'un langage (verbal ou non verbal) qui la signifie explicitement ou implicitement. Ce n'est que lorsqu'une règle est respectée ou transgressée qu'elle émerge de manière «concrète » ou « réelle », une fois que son résultat est observé. Un résultat qui peut aussi porter à conséquences en déclenchant l'activation de nouvelles règles telles que les sanctions et les gratifications ...

Mais les règles ne peuvent pas être directement observées. Si d'un point de vue structuraliste, nous cherchons à représenter cet ensemble de règles en prenant soin de reconstituer sa structure, il s'agit bel et bien selon la formule de Umberto Eco (1984) d'une structure abstraite ou «absente». Concrètement, ce n'est qu'une fois que les faits sont réalisés et observés comme tels en posant comme hypothèse qu'ils sont nécessairement déterminés par des règles sociales reliées systématiquement entre elles, que l'on peut tenter de formuler cet ensemble de règles abstraites en tant que «modèle », et faire apparaître leurs relations systématiques.

Pour rendre compte systématiquement de leurs déterminismes sur les pratiques, il convient de se doter de moyens conceptuels suffisamment précis pour discerner, identifier, analyser et déduire les mécanismes que ces règles sous-tendent.

Or ces moyens, les logiques modales en fournissent déjà de très importants, particulièrement grâce à la logique déontique de G. H. Von Wright, qui depuis son célèbre texte de 1951, a connu d'amples développements. Les travaux de certains auteurs comme Kalinowski ou Bailhache, ne concernent pas uniquement les sciences juridiques et quelques autres disciplines, mais particulièrement l'anthropologie dans son ensemble, de l'anthropologie juridique à l'anthropologie de la parole, en passant par l'anthropologie économique.

Pour le montrer, nous entrerons tout de suite dans le vif du sujet en rappelant les définitions utilisées en logique pour la modalité ontique et la modalité déontique. Plutôt que de remettre en cause ces définitions dans le cadre d'un emploi anthropologique, on peut penser que celles-ci sont tout à fait pertinentes, à condition d'être précisées pour pouvoir s'adapter à la diversité des contextes socioculturels, et d'être complétées par d'autres modalités, notamment la modalité axiologique.

Après avoir présenté ces règles, nous testerons la pertinence anthropologique de leurs définitions en les utilisant pour décrire les déterminismes institutionnels de l'organisation sociale. En prenant le cas de la société yucuna d'Amazonie colombienne dont les règles ont déjà fait l'objet de notre thèse (Fontaine, 2001), nous montrerons qu'une analyse en termes de logiques modales révèle les mécanismes d'un système social à différents niveaux. D'abord, en permettant la comparaison entre plusieurs de ces systèmes, ce qui ouvre le champ à des analyses socio-économiques et historiques. Ensuite, en éclairant les conditions institutionnelles de reconnaissance des catégories, ce qui explicite les «conditions de félicité » des actes performatifs soulevées par Austin et Searle. Enfin, en articulant dans un même système théorique les langages et les actions d'une société donnée, ce qui permet d'expliquer le fonctionnement des interactions verbales et non verbales dans des contextes variés.

\section{Les règles en termes de logiques modales}

Les règles ontiques

Rappelons que la logique ontique (ou aléthique) est une logique modale dont les principes fondamentaux ont été posés par Aristote. Sans chercher ici en faire une présentation globale, nous n'indiquerons que les équivalences du carré d'Aristote synthétisées en 1966 par Robert Blanché.

Nécessaire $=$ Non contingent $=$ Impossible de ne pas $=$ Non possible de ne pas

Possible $=$ Non impossible $=$ Contingent de ne pas $=$ Non nécessaire de ne pas 
Impossible $=$ Non possible $=$ Nécessaire de ne pas $=$ Non contingent de ne pas

Contingent $=$ Non nécessaire $=$ Possible de ne pas $=$ Non impossible de ne pas

Nous reprendrons la notation courante suivante :

$\square:$ Nécessaire

$\diamond$ : Possible

$\sim$ : Négation

$\Rightarrow$ : Implication

$\&$ : et (conjonction)

$\checkmark$ : ou non exclusif, au moins l'un (disjonction).

W : ou exclusif $(l u \ll$ Soit ..., soit ... »).

Certaines équivalences se notent alors ainsi :

$\square=\sim \diamond \sim \quad($ lu « Nécessaire $=$ Non possible de ne pas »)

$\diamond=\sim \square \sim \quad(\mathrm{lu} \ll$ Possible $=$ Non nécessaire de ne pas $»)$

Parmi les autres tautologies les plus utilisées, nous avons avec p et q, deux événements quelconques :

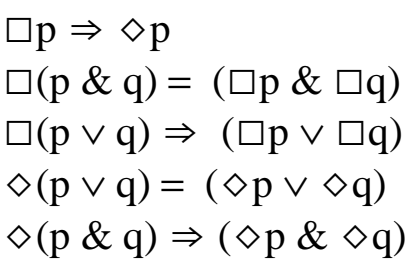

\section{Les règles déontiques}

Pour analyser différentes règles, il est tout à fait intéressant de tester la distinction effectuée en logique déontique entre quatre types de règle, dont deux positives: l'obligatoire (noté $\mathrm{O}$ ) et le permis (noté $\mathrm{P}$ ), et deux négatives : l'interdit (noté $\mathrm{I}$ ) et le facultatif (noté F). Rappelons que depuis les travaux de G. H. Von Wright (1951), les logiciens de la déontique reconnaissent différentes tautologies marquées par des équivalences et des implications (voir entre autres Kalinowski, 1996 : 107-108 ; Bailhache, 1991 : 14-15).

En ce qui concerne les équivalences les plus simples, nous avons :

Obligatoire $=$ Non facultatif $=$ Interdit de ne pas $=$ Non permis de ne pas

Permis $=$ Non interdit $=$ Facultatif de ne pas $=$ Non obligatoire de ne pas

Interdit $=$ Non permis $=$ Obligatoire de ne pas $=$ Non facultatif de ne pas

Facultatif $=$ Non obligatoire $=$ Permis de ne pas $=$ Non interdit de ne pas

Ce qui peut également se noter :

$\mathrm{O}=\sim \mathrm{F}=\mathrm{I} \sim=\sim \mathrm{P} \sim$

$\mathrm{P}=\sim \mathrm{I}=\mathrm{F} \sim=\sim \mathrm{O} \sim$

$\mathrm{I}=\sim \mathrm{P}=\mathrm{O} \sim=\sim \mathrm{F} \sim$

$\mathrm{F}=\sim \mathrm{O}=\mathrm{P} \sim=\sim \mathrm{I} \sim$

Parmi les autres tautologies les plus utilisées, nous avons :

$\mathrm{O} \Rightarrow \mathrm{P} \quad$ (lu «l'obligation implique le permis »)

$\mathrm{O}(\mathrm{p} \& \mathrm{q})=(\mathrm{Op} \& \mathrm{Oq})$

$\mathrm{O}(\mathrm{p} \vee \mathrm{q}) \Rightarrow(\mathrm{Op} \vee \mathrm{Oq})$

$P(p \vee q)=(P p \vee P q)$ 
$\mathrm{P}(\mathrm{p} \& \mathrm{q}) \Rightarrow(\mathrm{Pp} \& \mathrm{Pq})$

Mais d'un point de vue anthropologique, ces seules règles déontiques ne sont pas suffisantes pour décrire ou expliquer le fonctionnement des règles d'une société donnée, en raison soit de leur surdétermination, soit de leur indétermination. En effet, tout dans une société n'est pas déterminé uniquement par des obligations ou des interdits. Mais dans ce cas, qu'est-ce qui pousse les sujets à réaliser ce qui serait uniquement permis ou facultatif ?

Selon nous, l'examen approfondi des règles sociales conduit alors à considérer des règles moins radicales, mais néanmoins opératoires : il s'agit des règles axiologiques.

Malheureusement, personne n'a, à notre connaissance, permis de faire connaître un système logique fondé sur de telles règles. C'est pourquoi nous avons dû tenter de les définir.

\section{Les règles axiologiques}

Une seconde manière de s'intéresser aux règles consiste à préciser leur valeur, ce qui les place dans le cadre de la modalité axiologique.

Les règles axiologiques évaluent les différentes manières d'agir ou de non agir, d'être ou de ne pas être. Nous appelons le valorisé (noté V), tout ce qui a une valeur positive, et le dévalorisé (noté $\mathrm{D}$ ), tout ce qui a une valeur négative. A la différence des règles déontiques, nous n'avons plus d'oppositions (disjonctions inclusives ou exclusives), mais simplement des contrastes de degré entre le fortement valorisé et le faiblement valorisé, le fortement dévalorisé et le faiblement dévalorisé. Ces règles se définissent par rapport à des limites en terme de valorisation. Parmi celles-ci, certaines règles estiment des valeurs maximums et minimums.

Nous appellerons l'idéal ou le normé (noté $\mathrm{N}$ ), ce qui est le plus valorisé, et l'antinormé (noté $\mathrm{U}$ ), ce qui est le plus dévalorisé ou abject. Théoriquement, on peut attribuer un indice compris entre - 1 et 1 pour mesurer l'appréciation donnée par les règles axiologiques. On parlera de valorisation, si l'indice est inférieur ou égal à 1 et supérieur à 0 , de dévalorisation, si l'indice est supérieur ou égal à -1 et inférieur à 0 , de normé, si l'indice est égal à 1 , d'anti-normé s'il est égal à -1, et d'a-valorisé (noté $\Phi$ ), si l'indice est égal à 0 .

A-valorisation signifie la neutralité absolue en matière de règle, il signifie l'absence de toute prescription ou proscription. L'acte n'est régi par aucune règle axiologique ou déontique.

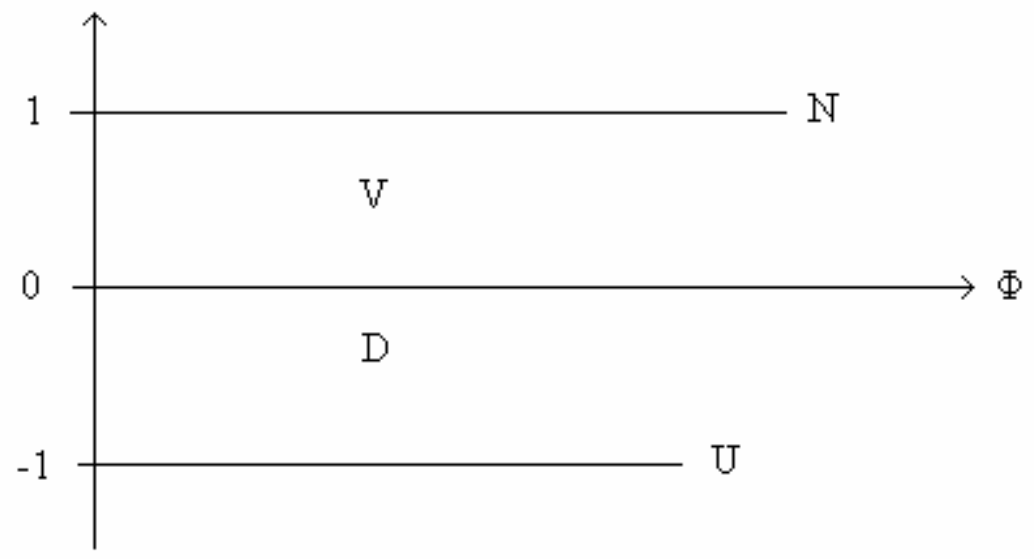

Notre objectif n'étant pas ici d'indiquer toutes les tautologies de ce modèle, nous n'exposerons que les plus utiles et les plus évidentes :

Valorisé $=$ [Non a-valorisé \& non dévalorisé $]=$ Non[A-valorisé $\vee$ dévalorisé $]$ 
Dévalorisé $=$ [Non a-valorisé \& non valorisé $]=$ Non[A-valorisé $\vee$ valorisé $]$

A-valorisé $=$ [Non valorisé \& non dévalorisé $]=$ Non[valorisé $\vee$ dévalorisé $]$

Non valorisé $=[$ A-valorisé $\vee$ dévalorisé $]$

Non dévalorisé $=[A$-valorisé $\vee$ valorisé $]$

Ce qui peut aussi se noter :

$\mathrm{V}=[\sim \Phi \& \sim \mathrm{D}]=\sim[\Phi \vee \mathrm{D}]$

$\mathrm{D}=[\sim \Phi \& \sim \mathrm{V}]=\sim[\Phi \vee \mathrm{V}]$

$\Phi=[\sim \mathrm{V} \& \sim \mathrm{D}]=\sim[\mathrm{V} \vee \mathrm{D}]$

$\sim \mathrm{V}=[\Phi \vee \mathrm{D}]$

$\sim \mathrm{D}=[\Phi \vee \mathrm{V}]$

Nous avons aussi :

$\mathrm{Np} \Rightarrow \mathrm{Vp}$
$\mathrm{Up} \Rightarrow \mathrm{Dp}$
$\mathrm{Vp} \Rightarrow \mathrm{D} \sim \mathrm{p}$
$\mathrm{Dp} \Rightarrow \mathrm{V} \sim \mathrm{p}$
$\mathrm{N}(\mathrm{p} \& \mathrm{q})=(\mathrm{Np} \& \mathrm{Nq})$
$\mathrm{N}(\mathrm{p} \vee \mathrm{q}) \Rightarrow(\mathrm{Np} \vee \mathrm{Nq})$
$\mathrm{V}(\mathrm{p} \& \mathrm{q})=(\mathrm{Vp} \& \mathrm{Vq})$
$\mathrm{V}(\mathrm{p} \vee \mathrm{q}) \Rightarrow(\mathrm{Vp} \vee \mathrm{Vq})$
$\mathrm{V} \Rightarrow \sim \Phi$
$\mathrm{D} \Rightarrow \sim \Phi$

\section{Le système social d'un point de vue sociologique et historique}

Dans cette partie, nous tenterons de donner une description formalisée de la structure traditionnelle de répartition des tâches, c'est-à-dire épurée des effets les plus importants de la modernité. En considérant que celle-ci est liée aux contacts directs de l'ensemble de la population indigène avec les Blancs, nous pouvons estimer que la transformation de cette structure débute avec l'arrivée des premiers caucheros au tout début du XXème siècle. Contrairement à celle que nous donnerons pour la structure contemporaine, la structure de répartition des tâches existant avant le XXème siècle ne peut évidemment pas être décrite précisément à partir d'observations directes, ni même de travaux de seconde mains puisque aucun ethnographe n'a fait d'enquête à ce sujet à cette époque. La structure que nous indiquons n'est qu'une estimation tirée essentiellement de la comparaison des données ethnographiques recueillies à partir de la fin des années soixante ${ }^{2}$ avec nos propres données actuelles.

\section{La répartition des tâches par sexe}

Comme dans la plupart des cultures natives d'Amazonie, les tâches des Yucuna sont d'abord distribuées en fonction du sexe. Certaines sont ainsi réservées aux femmes, d'autres aux hommes. Traditionnellement, beaucoup de ces tâches ne sont pas simplement «valorisées » ou «idéales », elles incombent à chacun en fonction de son genre masculin ou féminin.

\footnotetext{
${ }^{2}$ Cette comparaison doit beaucoup aux informations de P.-Y. Jacopin présent sur le terrain à cette époque.
} 
Selon l'équivalence notée plus haut, dire qu'elles sont «obligatoires » équivaut à dire qu'il est «interdit de ne pas les faire ». Ne pas réaliser les tâches qui marquent la virilité (chasser, travailler le bois, etc.) quand on est né de sexe masculin, revient à être exclu de la catégorie «homme » (achiñá en yucuna) et donc de ne pas être traité comme tel. De même, pour une femme (inanaru), ne pas cultiver les tubercules est impensable du point de vue traditionnel.

La répartition sexuelle des tâches peut alors être analysée en terme de règles qui peuvent être au moins en partie identifiées comme des obligations. Bien entendu, cette répartition sexuelle des tâches n'est pas uniquement réductible à des obligations, nous verrons plus loin quels autres types de règles, elle peut englober.

Notons donc formellement de quelle manière les obligations incombent aux femmes et aux hommes.

Les femmes (notées Fe) sont obligées de cultiver des tubercules (manioc, igname, taro, patate douce), de préparer les aliments, de fabriquer les poteries, d'élever les enfants, etc.

$\mathrm{R} 1=\mathrm{O}\{\mathrm{Fe}$, Cultiver, Tubercule $\}$

$\mathrm{R} 2=\mathrm{O}\{\mathrm{Fe}$, Préparer, Aliment $\}$

$\mathrm{R} 3=\mathrm{O}\{\mathrm{Fe}$, Fabriquer, Poterie $\}$

$\mathrm{R} 4=\mathrm{O}\{\mathrm{Fe}$, Elever, Enfant $\}$

$\mathrm{R} 5=\mathrm{O}\{\mathrm{Fe}$, Laver \& Nettoyer, Maison \& Bien $\}$

Les noms d'ensembles notés en italiques sont des sous-ensembles quelconques de ces ensembles. Tubercule signifie « un ou des tubercules quelconques » et non pas « les (ou tous les) tubercules ». Par contre l'obligation est valable pour «(toutes) les femmes » (de la société yucuna), c'est pourquoi Fe n'est pas en italique.

Les hommes (notés Ho) sont obligés de chasser, pêcher, fabriquer les vanneries (paniers, plats, égouttoirs, tamis, nasses, etc.), travailler le bois (canoë, banc, pilon, etc.), de semer et préparer les aliments spirituels ou rituels (coca, tabac, ananas). Ils sont également obligés d'essarter, d'édifier des maisons et de tresser des toitures de feuilles.

R6 $=\mathrm{O}\{$ Ho, Tuer, Gibier $\}$

$\mathrm{R} 7=\mathrm{O}\{$ Ho, Pêcher, Poisson $\}$

$\mathrm{R} 8=\mathrm{O}\{$ Ho, Fabriquer, Vannerie $\}$

$\mathrm{R} 9=\mathrm{O}\{$ Ho, Travailler, Bois $\}$

$\mathrm{R} 10=\mathrm{O}\{\mathrm{Ho}$, Semer \& Préparer, Coca $\}$

$\mathrm{R} 11=\mathrm{O}\{\mathrm{Ho}$, Semer \& Préparer, Tabac $\}$

$\mathrm{R} 12=\mathrm{O}\{$ Ho, Semer \& Préparer, Ananas $\}$

$\mathrm{R} 13=\mathrm{O}\{$ Ho, Essarter, Jardin $\}$

$\mathrm{R} 14=\mathrm{O}\{$ Ho, Edifier, Maison $\}$

$\mathrm{R} 15=\mathrm{O}\{\mathrm{Ho}$, Tresser, Toiture_de_feuilles $\}$

Signalons que nous ne considérons ici que des hommes et des femmes âgés de 20 à 49 ans. Nous verrons plus loin ce qu'il en est des autres classes d'âge.

Pour l'instant, nous n'entrons pas davantage dans le détail des activités, ni dans la transformation des objets à laquelle elles aboutissent, pour prendre le temps d'assimiler cette notation formelle. Indiquons simplement que l'ordre syntaxique de base prend la forme :

Règle $\{$ sujet(s), verbe à l'infinitif, complément(s), temps, lieu, aspect $\}$

Remarquons d'ailleurs que cette répartition des tâches entre les hommes et les 
femmes suppose non seulement des obligations pour tel ou tel sexe, mais également d'autres règles pour le sexe opposé.

Si la chasse est une obligation pour les hommes, il convient d'indiquer ce qu'il en est pour les femmes. Le rapport social entre hommes et femmes devient alors un rapport de règles pour un ensemble d'activités particulières.

Ce qui nous amène à décrire la structure sociale par une série de règles décomposables pour les hommes et les femmes. Dans ce cas, il est intéressant de comparer la distribution des règles pour chaque catégorie de sujet. Formellement, nous avons choisi de séparer l'ordonnancement de cette distribution avec $\backslash$

Par exemple, nous lirons la première règle $\mathrm{R} 1$ de la série qui suit de la manière suivante : «Il est obligatoire pour les femmes, et interdit pour les hommes, de cultiver des tubercules $»$.

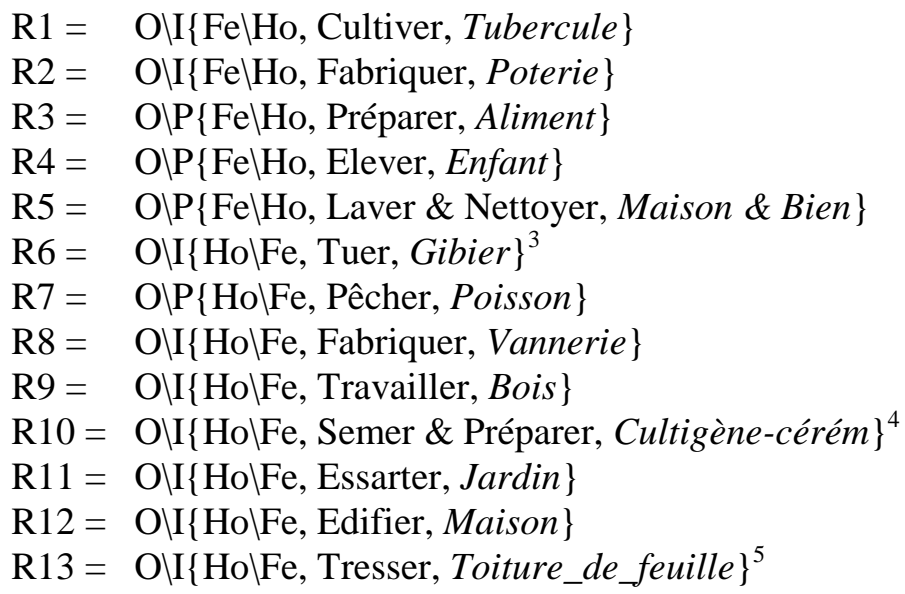

La chasse, la fabrication des vanneries, le travail du bois, l'essartage, l'édification des maisons, le travail du bois, semer et préparer les cultigènes cérémoniels, sont des obligations pour les hommes, et des interdits pour les femmes (du moins selon la répartition sociale traditionnelle, ce qui comme on le verra n'est plus nécessairement le cas aujourd'hui). Inversement, la culture des tubercules et la fabrication des poteries sont obligatoires pour les femmes, mais interdites aux hommes.

D'autres répartitions sexuelles des tâches sont plus souples. L'obligation de pêcher pour les hommes ne correspond pas à une interdiction pour les femmes. Celles-ci peuvent très bien pêcher pour survivre (avec des nasses ou tamis), si elles vivent seules ou en l'absence des hommes. Indiquons néanmoins que certaines techniques de pêche restent pour elles interdites, par exemple : écraser les racines piscicides (barbasco) lors de la pêche à la nivrée (mais elles participent à la prise des poissons). De même, l'obligation pour les femmes de préparer les aliments ou d'élever les enfants ne suppose pas que ces tâches soient interdites aux hommes. Ceux-ci pourront, et seront même obligés, de les accomplir en l'absence des femmes. Si l'on n'examine pas chaque contexte particulier ${ }^{6}$ pour préciser

\footnotetext{
3 Sous la catégorie « Gibier », nous entendons l'ensemble des mammifères et des oiseaux arboricoles, dont la chasse est traditionnellement réservée aux hommes. Pour se référer aux listes d'animaux et de plantes concernant les tâches réglementées, voir Fontaine 2001.

${ }^{4}$ Les cultigènes cérémoniels sont la coca, le tabac, l'ananas, l'achiote et les maracas (Couraupita guianensis). L'ensemble des cultigènes cérémoniels est noté Culti-cérém, car le tiret introduit un sous ensemble «les cultigènes cérémoniels inclus dans l'ensemble des cultigènes ».

5 A la différence du tiret court, le tiret en bas n'introduit pas un sous-ensemble. Il s'agit d'un même ensemble portant, à défaut d'analyse propositionnelle plus complexe, une étiquète à plusieurs mots.

${ }_{6}^{6}$ Au premier stade de cette présentation qui se situe au niveau des règles les plus générales de l'ensemble de
} 
s'il est réglementé ou non par cette obligation, on est contraint de se donner une règle générale plus faible (ou moins déterminante), qui sera dans ce cas une simple permission.

Une fois utilisée la typologie de la logique déontique pour classer les règles qui structurent la répartition sexuelle des tâches, celles-ci se montrent évidemment loin d'être suffisantes pour expliquer le fonctionnement de l'organisation sociale ou les comportements des sujets.

Si l'on comprend aisément que les obligations et les interdits déterminent les actes dans le jeu des pressions ou des contraintes sociales (qu'il reste bien entendu à analyser en détail avec chaque situation particulière), peut-on pour autant en déduire que tous les actes soient influencés par de telles règles?

Et si l'on considère des règles aussi peu contraignantes que le permis et le facultatif, celles-ci ne sont pas suffisantes pour décider les sujets sociaux à réaliser les actes qu'elles autorisent. Et cela parce que la logique déontique pose toujours la possibilité ou non de la violation de la règle, et par conséquent de la sanction. L'obligation et l'interdiction peuvent être violées et donc sanctionnées, alors que le permis et le facultatif ne le peuvent pas.

On peut alors douter que l'alternative sanction \non-sanction permette d'envisager tous les actes sociaux. Ce qui équivaut à dire que la modalité déontique n'apparaît pas suffisante pour considérer l'ensemble des règles sociales, qui au sein d'un système social donné, déterminent les activités des sujets.

C'est alors qu'il est nécessaire de faire appel à la modalité axiologique en distinguant le valorisé, le normé, le dévalorisé et l'anti-normé.

Ces règles ont l'avantage d'affiner davantage les formules censées rendre compte de la répartition des tâches.

On peut alors considérer :

R7.1 = OIV $\{\mathrm{HolFe}$, Pêcher, Poisson $\}$

Dans le cadre d'une description générale, $\mathrm{O} \backslash \mathrm{P}\{\mathrm{HolFe}$, Pêcher, Poisson $\}$ peut être remplacé par $\mathrm{O} \backslash \mathrm{V}\{\mathrm{HolFe}$, Pêcher, Poisson $\}$, car il est toujours valorisant pour une femme de savoir pêcher (du moins selon les techniques qui lui sont permises).

Nous pouvons aussi ajouter :

$\mathrm{R} 14=\mathrm{O} \backslash \mathrm{V}\{\mathrm{Ho} \backslash \mathrm{Fe}$, Porter \& Couper, Bois_de_feu $\}$

$\mathrm{R} 15=\mathrm{O} \backslash \mathrm{V}\{\mathrm{HolFe}$, Cueillir, Fruit-sylvestre $\}$

$\mathrm{R} 16=\mathrm{O} I \mathrm{~V}\{\mathrm{HolFe}$, Tuer, Insecte-comestible \& Tortue-terrestre \& Oiseau-terrestre $\}$

$\mathrm{R} 17=\mathrm{V} \backslash\{$ HolFe, Cultiver, Barbasco $\}$

S'il est vrai que le port et la coupe du bois sont obligatoires pour les hommes, on ne peut pas dire pour les femmes qu'elles sont interdites. Il est d'ailleurs insuffisant de dire qu'elles sont permises ou facultatives, car dans ce cas, qu'est-ce qui les motive ?

Dans certains cas, il convient de laisser $\mathrm{P}$, sans le remplacer par $\mathrm{V}$, car il est parfois difficile de généraliser une valorisation. Ainsi, les hommes ne valorisent pas toujours la préparation des aliments, l'élevage des enfants et le nettoyage. Le plus souvent, ils le font

la société, nous ne traitons pas des contextes particuliers. En fait, l'étude des règles accomplies devient de plus en plus complexe à mesure que la liste des règles s'allonge, et que celles-ci se confortent, se conditionnent ou entrent en contradiction les unes par rapport aux autres. Les analyses séquentielles de situations d'interaction verbales et non verbales font apparaître cette complexité (Fontaine, 2001). 
lorsqu'ils y sont contraints, en l'absence des femmes. Nous ne touchons donc pas aux trois formules suivantes :

$\mathrm{R} 3=\mathrm{O} \backslash \mathrm{P}\{\mathrm{Fe} \backslash \mathrm{Ho}$, Préparer, Aliment $\}$

$\mathrm{R} 4=\mathrm{O} \backslash \mathrm{P}\{\mathrm{Fe} \backslash \mathrm{Ho}$, Elever, Enfant $\}$

$\mathrm{R} 5=\mathrm{O} \backslash \mathrm{P}\{\mathrm{Fe} \backslash \mathrm{Ho}$, Laver \& Nettoyer, Maison \& Biens $\}$

On voit bien qu'un tel cadre général est loin d'être suffisant pour expliquer les fonctionnements particuliers. Même s'il a le mérite de dresser un aperçu global des règles en vigueur, on ne saurait se passer d'identifications et d'analyses plus précises en situation, notamment dans les dynamiques interactionnelles ou interlocutives.

\section{La répartition des tâches par âge}

Une autre répartition fondamentale est celle qui divise les enfants (yuwajî), les adolescents (walijímina), les adultes (pheñawilá) et les vieux (phíyu'ke). En dehors de ces classes d'âges, il y a bien sûr les enfants en bas âge (to'jmá). Mais personne ne leur donne de tâches à accomplir si bien qu'ils sont toujours à la charge des trois autres classes d'âge.

Nous poserons la notation suivante :

$\mathrm{S}_{20-49}$ : Ensemble des sujets de 20 à 49 ans.

$\mathrm{F}_{50+}$ : Ensemble des sujets de sexe féminin de plus de 50 ans.

$\mathrm{H}_{20-49}$ : Ensemble des sujets de sexe masculin de 20 à 49 ans.

$\mathrm{F}_{5-14}$ : Ensemble des sujets de sexe féminin de 5 à 14 ans.

$\mathrm{H}_{0-4}$ : Ensemble des sujets de sexe masculin de moins de 5 ans.

$\mathrm{S}_{5-19} \cap \mathrm{S}_{50+}$ : Ensemble des sujets de 5 à 19 ans et de plus de 50 ans.

En considérant ces nouveaux sous-ensembles par classe d'âge, l'on peut préciser la structure de certaines tâches n'étant pas réparties entre sexes, mais uniquement selon les catégories d'âges.

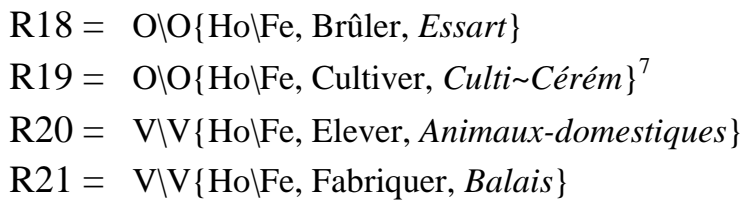

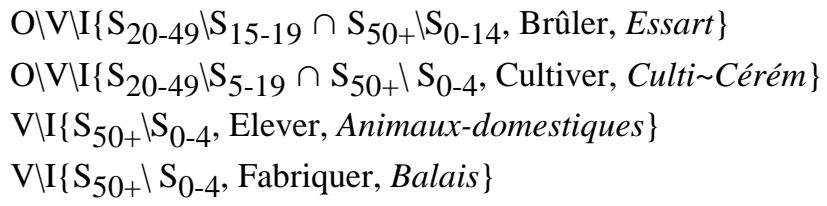

La répartition par catégories d'âge peut être détaillée dans le tableau suivant :

\begin{tabular}{|c|c|c|c|c|c|c|c|c|c|c|c|}
\hline Sexe & \multicolumn{5}{|c|}{ Homme } & \multicolumn{5}{|c|}{ Femme } & \\
\hline Age & $0-4$ & $5-14$ & $15-19$ & $20-49$ & $50+$ & $50+$ & $20-49$ & $15-19$ & $5-14$ & $0-4$ & Notation des activités \\
\hline R1 & $\mathrm{I}$ & $\mathrm{I}$ & I & $\mathrm{I}$ & $\mathrm{I}$ & $\mathrm{O}$ & $\mathrm{O}$ & $\mathrm{O}$ & $\mathrm{V}$ & $\mathrm{I}$ & Cultiver, Tubercule \\
\hline R2 & I & I & $\mathrm{P}$ & $\mathrm{P}$ & $\mathrm{P}$ & $\mathrm{O}$ & $\mathrm{O}$ & $\mathrm{O}$ & $\mathrm{V}$ & I & Préparer, Aliment \\
\hline R3 & I & I & I & I & I & $\mathrm{O}$ & $\mathrm{O}$ & $\mathrm{O}$ & $\mathrm{V}$ & I & Fabriquer, Poterie \\
\hline $\mathrm{R} 4$ & $\mathrm{I}$ & $\mathrm{O}$ & $\mathrm{P}$ & $\mathrm{P}$ & $\mathrm{V}$ & $\mathrm{O}$ & $\mathrm{O}$ & $\mathrm{O}$ & $\mathrm{O}$ & I & Elever, Enfant \\
\hline R5 & $\mathrm{I}$ & $\mathrm{I}$ & $\mathrm{V}$ & $\mathrm{O}$ & $\mathrm{V}$ & $\mathrm{I}$ & $\mathrm{I}$ & $\mathrm{I}$ & $\mathrm{I}$ & I & Tuer, Gibier \\
\hline R6 & I & $\mathrm{P}$ & $\mathrm{P}$ & $\mathrm{P}$ & $\mathrm{P}$ & $\mathrm{O}$ & $\mathrm{O}$ & $\mathrm{O}$ & $\mathrm{P}$ & I & $\begin{array}{l}\text { Laver \& Nettoyer, Maison \& } \\
\text { Biens }\end{array}$ \\
\hline $\mathrm{R} 7$ & $\mathrm{I}$ & $\mathrm{V}$ & $\mathrm{O}$ & $\mathrm{O}$ & $\mathrm{V}$ & $\mathrm{V}$ & $\mathrm{V}$ & $\mathrm{V}$ & $\mathrm{V}$ & $\mathrm{I}$ & Pêcher, Poisson \\
\hline $\mathrm{R} 8$ & $\mathrm{I}$ & $\mathrm{V}$ & $\mathrm{O}$ & $\mathrm{O}$ & $\mathrm{O}$ & $\mathrm{I}$ & I & $\mathrm{I}$ & I & $\mathrm{I}$ & Fabriquer, Vannerie \\
\hline R9 & I & $\mathrm{V}$ & $\mathrm{O}$ & $\mathrm{O}$ & V & I & I & $\mathrm{I}$ & I & I & Travailler, Bois \\
\hline
\end{tabular}

\footnotetext{
${ }^{7}$ Culti cérém désigne les cultigènes non cérémoniels. Culti cérém = Anón, Avocat, Banane, banane plantain, Caimito, Canne à sucre, Echalotte, Guamo, Guayaba, Lulo, Maïs, Mangue, Marañon, Papaye, Poma rosa, Uva., Piment (non chamanique).
} 


\begin{tabular}{|l|l|l|l|l|l|l|l|l|l|l|l|}
\hline R10 & I & V & O & O & O & I & I & I & I & I & Semer \& Préparer, Culti-cérém \\
\hline R11 & I & I & O & O & V & I & I & I & I & I & Essarter, Jardin \\
\hline R12 & I & V & O & O & V & I & I & I & I & I & Edifier, Maison \\
\hline R13 & I & V & O & O & O & I & I & I & I & I & Tresser, Toiture \\
\hline R14 & I & V & O & O & V & V & V & V & I & I & Porter \& Couper, Bois_de_feu \\
\hline R15 & I & V & O & O & V & V & V & V & V & I & Cueillir, Fruit-sylvestre \\
\hline R16 & I & V & O & O & O & V & V & V & V & I & $\begin{array}{l}\text { Tuer, Insecte-comestible \& Tortue- } \\
\text { terrestre \& Oiseau-terrestre }\end{array}$ \\
\hline R17 & I & I & V & V & V & I & I & I & I & I & Cultiver, Barbasco \\
\hline R18 & I & I & V & O & V & V & O & V & I & I & Brûler, Essart \\
\hline R19 & I & V & V & O & V & V & O & V & V & I & Cultiver, Culticérém \\
\hline R20 & I & V & V & V & V & V & V & V & V & I & Elever, Animaux-domestiques \\
\hline R21 & I & V & V & V & V & V & V & V & V & I & Fabriquer, Balais \\
\hline
\end{tabular}

Pour évaluer la répartition relative des règles entre sexes et classes d'âge, il est toujours intéressant d'indiquer leur nombre dans un tableau.

\begin{tabular}{|c|c|c|c|c|c|c|c|c|c|c|c|c|c|c|}
\hline Age & \multicolumn{2}{|c|}{$0-4$} & \multicolumn{2}{|c|}{$5-14$} & \multicolumn{1}{|c|}{$15-19$} & $20-49$ & \multicolumn{2}{|c|}{$50+$} & \multicolumn{3}{|c|}{$0+$} \\
\hline Sexe & H & F & H & F & H & F & H & F & H & F & H & F & Indice \\
& & & & & & & & & & & & & \\
\hline O & 0 & 0 & 1 & 1 & 10 & 5 & 13 & 7 & 4 & 5 & 28 & 18 & 1,55 \\
\hline I & 21 & 21 & 7 & 10 & 2 & 8 & 2 & 8 & 2 & 8 & 34 & 55 & 0,61 \\
\hline V & 0 & 0 & 12 & 9 & 6 & 8 & 3 & 6 & 13 & 8 & 34 & 31 & 1,09 \\
\hline P & 0 & 0 & 1 & 1 & 3 & 0 & 3 & 0 & 2 & 0 & 9 & 1 & 9 \\
\hline Total & 21 & 21 & 21 & 21 & 21 & 21 & 21 & 21 & 21 & 21 & 10 & 105 & 1 \\
& & & & & & & & & & & 5 & & \\
\hline
\end{tabular}

Ce tableau offre le constat immédiat que les hommes sont toutes catégories d'âge confondues 1, 55 fois plus obligés que les femmes (28 obligations masculines contre 21 féminines), mais qu'ils subissent beaucoup moins d'interdits (34 pour les hommes contre 55 pour les femmes).

On remarque aussi que les obligations sont presque deux fois plus nombreuses pour les hommes que pour les femmes avant 50 ans ; alors qu'elles sont un peu plus élevées pour les femmes en fin de vie. Quant aux interdits, l'écart s'accentue particulièrement après 15 ans pour rester quatre fois plus élevé chez les femmes que chez les hommes.

Les activités valorisantes sont globalement un peu plus élevées chez les hommes. Elles sont nettement plus importantes pour eux en début et en fin d'activité (5-14 ans et plus de 50 ans), alors qu'on en compte davantage pour les femmes durant leur période de fécondité (15-49 ans). Enfin, certains droits des hommes (permissions) apparaissent 9 fois plus élevés que ceux des femmes ! S'ils sont équilibrés avant 15 ans, les femmes n'ont plus aucun droit spécifique après cet âge.

\section{La répartition des tâches aujourd'hui}

Si nous comparons les règles de la structure sociale traditionnelle à celle dont les applications s'observent encore aujourd'hui, nous trouvons de notables changements qu'il convient d'indiquer précisément.

En reprenant la répartition par sexe et par âge de la structure contemporaine, nous pouvons la comparer à la répartition contemporaine de la manière suivante :

\begin{tabular}{|l|l|}
\hline Répartition traditionnelle & Répartition contemporaine \\
\hline $\mathrm{R} 1=\mathrm{O} \backslash \mathrm{V} \backslash \mathrm{I}\left\{\mathrm{F}_{15+} \backslash \mathrm{F}_{5-14} \backslash \mathrm{H}_{0+} \cap \mathrm{F}_{0-4}\right.$, Cultiver, & $\mathrm{V} \backslash \mathrm{P} \backslash\left\{\mathrm{F}_{5+} \backslash \mathrm{H}_{5+} \backslash \mathrm{S}_{0-4}\right.$, Cultiver, Tubercule $\}$ \\
\hline
\end{tabular}




\begin{tabular}{|c|c|}
\hline Tubercule\} & \\
\hline $\begin{array}{l}\mathrm{R} 2=\mathrm{O} \backslash \mathrm{V} \backslash \mathrm{P} \backslash\left\{\mathrm{F}_{15+} \backslash \mathrm{F}_{5-14} \backslash \mathrm{H}_{15+} \backslash \mathrm{H}_{0-14} \cap \mathrm{F}_{0-4},\right. \\
\text { Préparer, Aliment }\}\end{array}$ & $\mathrm{V} \backslash \mathrm{PUI}\left\{\mathrm{F}_{5+} \backslash \mathrm{H}_{5+} \backslash \mathrm{S}_{0-4}\right.$, Préparer, Aliment $\}$ \\
\hline $\begin{array}{l}\mathrm{R} 3=\mathrm{O} \backslash \mathrm{V} \backslash \mathrm{I}\left\{\mathrm{F}_{15+}{ } \mathrm{F}_{5-14} \backslash \mathrm{H}_{0+} \cap \mathrm{F}_{0-4}, \text { Fabriquer, }\right. \\
\text { Poterie }\}\end{array}$ & $\mathrm{VII}\left\{\mathrm{F}_{5+} \backslash \mathrm{H}_{0+} \cap \mathrm{F}_{0-4}\right.$, Fabriquer, Poterie $\}$ \\
\hline $\begin{array}{l}\mathrm{R} 4=\mathrm{O} \backslash \mathrm{V} \backslash \mathrm{P} \backslash\left\{\mathrm{F}_{5+} \cap \mathrm{H}_{5-14} \backslash \mathrm{H}_{50+} \backslash \mathrm{H}_{15-49} \backslash \mathrm{S}_{0-4},\right. \\
\text { Elever, Enfant }\}\end{array}$ & $\begin{array}{l}\mathrm{V} \backslash \mathrm{P} \backslash\left\{\mathrm{F}_{5+} \cap \mathrm{H}_{5-14} \cap \mathrm{H}_{50+} \backslash \mathrm{H}_{15-49} \backslash \mathrm{S}_{0-4}, \text { Elever, }\right. \\
\text { Enfant }\}\end{array}$ \\
\hline $\begin{array}{l}\mathrm{R} 5=\text { OIVII }\left\{\mathrm{H}_{20-49} \backslash \mathrm{H}_{15-19} \cap \mathrm{H}_{50+} \backslash \mathrm{H}_{0-14} \cap \mathrm{F}_{0+},\right. \\
\text { Tuer, Gibier }\}\end{array}$ & $\mathrm{V} \backslash \mathrm{I}\left\{\mathrm{H}_{15+} \backslash \mathrm{H}_{0-4} \cap \mathrm{F}_{0+}\right.$, Tuer, Gibier $\}$ \\
\hline $\begin{array}{l}\mathrm{R} 6=\mathrm{OIV \backslash P} \backslash\left\{\mathrm{F}_{15+} \backslash \mathrm{F}_{5-14} \backslash \mathrm{H}_{5+} \backslash \mathrm{S}_{0-4} \text {, Laver } \cap\right. \\
\text { Nettoyer, Maison } \cap \text { Bien }\}\end{array}$ & $\begin{array}{l}\mathrm{V} \backslash \mathrm{P \backslash I}\left\{\mathrm{F}_{5+} \backslash \mathrm{H}_{5+} \backslash \mathrm{S}_{0-4}, \text { Laver } \cap \text { Nettoyer, Maison } \cap\right. \\
\text { Bien }\}\end{array}$ \\
\hline $\begin{array}{l}\mathrm{R} 7=\mathrm{O} V \mathrm{~V} \backslash\left\{\mathrm{H}_{15-49} \backslash \mathrm{H}_{5-14} \cap \mathrm{H}_{50+} \cap \mathrm{F}_{5+} \backslash \mathrm{S}_{0-4},\right. \\
\text { Pêcher, Poisson }\}\end{array}$ & $\mathrm{VI}\left\{\mathrm{S}_{5+} \backslash \mathrm{S}_{0-4}\right.$, Pêcher, Poisson $\}$ \\
\hline $\begin{array}{l}\mathrm{R} 8=\mathrm{O} \backslash \mathrm{V} \backslash\left\{\mathrm{H}_{15+} \backslash \mathrm{H}_{5-14} \backslash \mathrm{H}_{0-4} \cap \mathrm{F}_{0+}, \text { Fabriquer, }\right. \\
\text { Vannerie }\}\end{array}$ & $\mathrm{V} \backslash \mathrm{P} \backslash\left\{\mathrm{H}_{15+} \backslash \mathrm{F}_{15+} \mid \mathrm{S}_{0-14}\right.$, Fabriquer, Vannerie $\}$ \\
\hline $\begin{array}{l}\mathrm{R} 9=\mathrm{O} \backslash \mathrm{V} \backslash\left\{\mathrm{H}_{15-49} \backslash \mathrm{H}_{5-14} \cap \mathrm{H}_{50+} \backslash \mathrm{H}_{0-4} \cap \mathrm{F}_{0+},\right. \\
\text { Travailler, Bois }\}\end{array}$ & $\mathrm{VIPI}\left\{\mathrm{H}_{5+} \backslash \mathrm{F}_{5+} \backslash \mathrm{S}_{0-4}\right.$, Travailler, Bois $\}$ \\
\hline $\begin{array}{l}\mathrm{R} 10=\mathrm{OIV \backslash I}\left\{\mathrm{H}_{15+} \backslash \mathrm{H}_{5-14} \backslash \mathrm{H}_{0-4} \cap \mathrm{F}_{0+}, \text { Semer } \cap\right. \\
\text { Préparer, Culti_cérém }\}\end{array}$ & $\begin{array}{l}\mathrm{V} \backslash \mathrm{P} \backslash\left\{\mathrm{H}_{15+} \cap \mathrm{F}_{50+} \backslash \mathrm{F}_{20-49} \backslash \mathrm{H}_{0-14} \cap \mathrm{F}_{0-19}, \text { Semer \& }\right. \\
\text { Préparer, Coca }\}\end{array}$ \\
\hline $\begin{array}{l}\mathrm{R} 11=\mathrm{O} V \mathrm{~V} \backslash\left\{\mathrm{H}_{15-49} \backslash \mathrm{H}_{50+} \backslash \mathrm{H}_{0-4} \cap \mathrm{F}_{0+}, \text { Essarter, }\right. \\
\text { Jardin }\}\end{array}$ & $\mathrm{VII}\left\{\mathrm{H}_{15+} \backslash \mathrm{H}_{0-14} \cap \mathrm{F}_{0+}\right.$, Essarter, Jardin $\}$ \\
\hline $\begin{array}{l}\mathrm{R} 12=\mathrm{O} V \backslash \mathrm{Y}\left\{\mathrm{H}_{15-49} \backslash \mathrm{H}_{5-14} \cap \mathrm{H}_{50+} \backslash \mathrm{H}_{0-4} \cap \mathrm{F}_{0+},\right. \\
\text { Edifier, Maison }\}\end{array}$ & $\mathrm{V} \backslash\left\{\mathrm{H}_{15+} \backslash \mathrm{H}_{0-14} \cap \mathrm{F}_{0+}\right.$, Edifier, Maison $\}$ \\
\hline $\begin{array}{l}\mathrm{R} 13=\mathrm{OIV \backslash I}\left\{\mathrm{H}_{15+} \backslash \mathrm{H}_{5-14} \backslash \mathrm{F}_{0+} \cap \mathrm{H}_{0-4}, \text { Tresser, }\right. \\
\text { Toiture }\}\end{array}$ & $\mathrm{VIP \backslash I}\left\{\mathrm{H}_{5+} \backslash \mathrm{F}_{15+} \backslash \mathrm{H}_{0-4} \cap \mathrm{F}_{0-14}\right.$, Tresser, Toiture $\}$ \\
\hline $\begin{array}{l}\mathrm{R} 14=\text { OIVII }\left\{\mathrm{H}_{15-49} \backslash \mathrm{H}_{5-14} \cap \mathrm{H}_{50+} \cap \mathrm{F}_{15+} \backslash \mathrm{H}_{0-}\right. \\
\left.4 \cap \mathrm{F}_{0-14} \text {, Porter \& Couper, Bois_de_feu }\right\}\end{array}$ & $\begin{array}{l}\mathrm{V} \backslash\left\{\mathrm{H}_{5+} \cap \mathrm{F}_{15+} \backslash \mathrm{H}_{0-4} \cap \mathrm{F}_{0-14} \text {, Porter \& Couper, }\right. \\
\text { Bois_de_feu }\}\end{array}$ \\
\hline $\begin{array}{l}\mathrm{R} 15=\mathrm{OIVII}\left\{\mathrm{H}_{15-49} \backslash \mathrm{H}_{5-14} \cap \mathrm{H}_{50+} \cap \mathrm{F}_{5+} \backslash \mathrm{S}_{0-4},\right. \\
\text { Cueillir, Fruit_sylvestre }\}\end{array}$ & $\mathrm{V} \backslash\left\{\mathrm{S}_{5+} \mid \mathrm{S}_{0-4}\right.$, Cueillir, Fruit_sylvestre $\}$ \\
\hline $\begin{array}{l}\mathrm{R} 16=\mathrm{O} \backslash \mathrm{V} \backslash \mathrm{I}\left\{\mathrm{H}_{15+} \backslash \mathrm{H}_{5-14} \cap \mathrm{F}_{5+} \backslash \mathrm{S}_{0-4}, \text { Tuer, }\right. \\
\text { Insecte_comestible } \cap \text { Tortue_terrestre } \cap \\
\text { Oiseau_terrestre }\}\end{array}$ & $\begin{array}{l}\mathrm{V} \backslash\left\{\mathrm{S}_{5+} \backslash \mathrm{S}_{0-4}, \text { Tuer, Insecte_comestible } \cap\right. \\
\text { Tortue_terrestre } \cap \text { Oiseau_terrestre }\}\end{array}$ \\
\hline $\mathrm{R} 17=\mathrm{V} \backslash\left\{\left\{\mathrm{H}_{15+} \backslash \mathrm{F}_{0+} \cap \mathrm{H}_{0-14}\right.\right.$, Cultiver, Barbasco $\}$ & $\mathrm{VII}\left\{\mathrm{H}_{15+} \backslash \mathrm{H}_{0-14} \cap \mathrm{F}_{0+}\right.$, Cultiver, Barbasco $\}$ \\
\hline $\begin{array}{l}\mathrm{R} 18=\mathrm{O} \backslash \mathrm{V} \backslash \mathrm{I}\left\{\mathrm{S}_{20-49} \backslash \mathrm{S}_{15-19} \cap \mathrm{S}_{50+} \mid \mathrm{S}_{0-14}, \text { Brûler, }\right. \\
\text { Essart }\}\end{array}$ & $\mathrm{VI}\left\{\mathrm{S}_{15+} \mathrm{S}_{0-14}\right.$, Brûler, Essart $\}$ \\
\hline $\begin{array}{l}\mathrm{R} 19=\mathrm{O} \backslash \mathrm{V} \backslash \mathrm{I}\left\{\mathrm{S}_{20-49} \mid \mathrm{S}_{5-19} \cap \mathrm{S}_{50+} \backslash \mathrm{S}_{0-4}, \text { Cultiver, }\right. \\
\text { Culti Cérém }\}\end{array}$ & $\mathrm{V} \backslash\left\{\mathrm{S}_{15+} \backslash \mathrm{S}_{0-14}\right.$, Cultiver, Culti $\sim$ Cérém $\}$ \\
\hline $\mathrm{R} 20=\mathrm{V} \backslash\left\{\mathrm{S}_{5+}{ } \mathrm{S}_{0-4}\right.$, Elever, Animaux_domestiques $\}$ & $\mathrm{V \backslash I}\left\{\mathrm{S}_{5+} \backslash \mathrm{S}_{0-4}\right.$, Elever, Animaux_domestiques $\}$ \\
\hline $\mathrm{R} 21=\mathrm{V} \backslash \mathrm{I}\left\{\mathrm{S}_{5+} \mid \mathrm{S}_{0-4}\right.$, Fabriquer, Balais $\}$ & $\mathrm{V} \backslash \mathrm{I}\left\{\mathrm{S}_{5+} \mid \mathrm{S}_{0-4}\right.$, Fabriquer, Balais $\}$ \\
\hline
\end{tabular}

On constate que par rapport à la structure d'origine, les obligations semblent avoir disparues (du moins à ce niveau global d'étude de l'organisation sociale). Elles se sont toutes rangées avec les valorisations. En effet, le mode de vie traditionnel n'étant plus obligatoire en raison des activités proposées ou imposées par les Blancs, chacun peut, en principe, choisir d'abandonner les tâches qui incombent à son sexe et à sa catégorie d'âge. Les risques de sanction sont considérablement amoindris en raison des droits que la société englobante se charge de faire respecter. En d'autres termes, une organisation sociale perd ses obligations de réaliser certaines activités, si la société dominante interdit ces obligations.

Par exemple, les femmes de plus de 15 ans peuvent aujourd'hui refuser de cultiver le manioc (ce qui était impensable autrefois) en allant travailler comme cuisinière chez un patron Blanc. Même si cela ne convient pas forcément à sa famille, elle ne sera pas 
forcément sanctionnée pour autant. La culture du manioc reste donc valorisée, mais n'est plus obligatoire.

On observe aussi que les interdits des règles $1,8,9,10$ et 13 , se sont scindés pour laisser place à des droits revenant à certaines catégories de sujets. De même que pour les obligations, la société dominante interdit aux Yucuna d'interdire. Parfois la société traditionnelle abandonne d'ailleurs ses vieux principes pour mieux s'adapter aux nouvelles valeurs de la modernité.

C'est toujours le cas de la culture du manioc : si elle reste interdite pour les enfants en bas âge (comme pour toute activité d'ailleurs), les hommes de plus de 15 ans s'autorisent parfois à semer et récolter du manioc pour les vendre (surtout s'ils sont célibataires ou veufs). De leur côté, les femmes fabriquent parfois des objets (vannerie, bois) autrefois réservés aux hommes, pour en tirer un bon prix en tant que produits artisanaux. En outre, elles tressent les feuilles de toiture ou préparent la coca, lorsque la main-d'œuvre masculine vient à manquer (souvent en raison du travail avec les Blancs).

\begin{tabular}{|c|c|c|c|c|c|c|c|c|c|c|c|c|c|c|}
\hline Age & \multicolumn{2}{|c|}{$0-4$} & \multicolumn{2}{|c|}{$5-14$} & \multicolumn{2}{|c|}{$15-19$} & \multicolumn{2}{c|}{$20-49$} & \multicolumn{2}{|c|}{$50+$} & \multicolumn{4}{c|}{$0+$} \\
\hline Sexe & H & F & H & F & H & F & H & F & H & F & H & F & Indice \\
\hline O & 0 & 0 & 0 & 0 & 0 & 0 & 0 & 0 & 0 & 0 & 0 & 0 & - \\
\hline I & 21 & 21 & 9 & 10 & 1 & 5 & 1 & 4 & 1 & 4 & 33 & 44 & 0,75 \\
\hline V & 0 & 0 & 9 & 10 & 16 & 13 & 16 & 13 & 17 & 14 & 58 & 50 & 1,16 \\
\hline P & 0 & 0 & 3 & 1 & 4 & 3 & 4 & 4 & 3 & 3 & 14 & 11 & 1,27 \\
\hline Total & 21 & 21 & 21 & 21 & 21 & 21 & 21 & 21 & 21 & 21 & 105 & 105 & 1 \\
\hline
\end{tabular}

Grâce à un nouveau tableau de répartition relative des règles, on remarque que le nombre d'interdit n'a quasiment pas bougé pour les hommes (de 34 à 33) mais qu'il a beaucoup diminué pour les femmes (de 55 à 44). En contre partie, certains droits des femmes ont pris une notable importance, surtout dès qu'elles arrivent à l'âge de se marier ou de quitter le foyer de leurs parents $(15$ ans $){ }^{8}$ D'autre part, si l'on considère l'effondrement des obligations au profit des valorisations, il est clair que l'organisation sociale traditionnelle s'est considérablement assouplie, même pour les hommes. ${ }^{9}$

\section{Les conditions institutionnelles de reconnaissance des catégories}

D'un point de vue logique, recevoir tel qualificatif revient à être classé dans une catégorie, c'est-à-dire à être inclus dans un ensemble. Pour cela, il faut non seulement satisfaire un certain nombre de règles, mais encore qu'un certain milieu social les ait reconnues ou attestées (par un langage), le plus souvent (mais pas toujours) en disant que le respect de ces règles a été observé ou prouvé.

C'est seulement une fois que cette première étape a été effectuée que le sujet est classé et peut être régi, donc sanctionné en tant que tel s'il ne respecte pas les règles spécifiques à la qualité qu'on lui a attribuée, et dont certaines reprennent les mêmes

\footnotetext{
${ }^{8}$ On peut se demander si le «droit» (la permission) de tresser des feuilles de toiture, de préparer la coca ou de vendre de l'artisanat est une « liberté ». Il est difficile de répondre de façon tranchée, mais on est tenté de dire que la diminution des interdictions soumises à sanctions, et l'élargissement de l'éventail des choix d'activité tend à accroître cette liberté. Certes, tout dépend également des valeurs partagées de la société. Si notre point de vue extérieur dévalorise de telles activités, elles nous apparaissent contraignantes.

${ }^{9}$ Ce qui ne suffit pas pour dire que les Yucuna sont aujourd'hui globalement plus «libres » qu'autrefois, car nous n'avons pas analysé le système de la société dominante, qui a lui aussi ses règles strictes.
} 
conditions que celles qui lui ont permis d'être qualifié ainsi.

Il convient donc de ne pas confondre les règles d'attribution d'un qualificatif, et les règles propres à celui-ci, une fois qu'il a été reconnu avec ce qualificatif, c'est pourquoi nous distinguons les règles qualificatoires des règles de fonctionnement.

\section{Les règles qualificatoires}

Par règle qualificatoire, nous entendons toute règle dont le respect est obligatoire, mais non obligatoirement suffisant pour qu'il soit permis d'attribuer à un sujet tel qualificatif. Elle équivaut à l'obligation pour un sujet de respecter une (ou plusieurs) règle(s) stricte(s) dont la sanction est l'interdiction de le qualifier avec tel qualificatif.

C'est pourquoi toute règle qualificatoire (ou classificatoire) est également une règle à sanction disqualificatoire. Sa violation est suffisante pour entraîner obligatoirement un interdit de qualification.

On peut donc dire que $\mathrm{R}$ est une règle qualificatoire, si la formule suivante est vérifiée : Il est obligatoire de permettre que l'on dise que $s$ est inclus dans telle catégorie, s'il a respecté telle(s) règle(s). Cette formule peut être notée :

$\mathrm{O}\{\mathrm{P}(\ll s \subset C t g »)$ si $\mathrm{R}(\mathrm{s}, \mathrm{Resp})\}$

Avec $C t g$ : une catégorie quelconque de sujet.

«... : Les guillemets pour marquer les limites de ce qui est énoncé.

Resp : Le marqueur de respect d'une règle.

si : Le marqueur de condition. ${ }^{10}$

Ce qui équivaut à : Il est obligatoire d'interdire que l'on dise que $s$ est inclus dans telle catégorie, s'il n'a pas respecté telle(s) règle(s).

$\mathrm{O}\{\mathrm{I}(\ll s \subset C t g »)$ si $\mathrm{R}(\mathrm{s}, \sim \operatorname{Resp})\}$

Ou encore à : Il est obligatoire que le respect de telle(s) règle(s) implique l'interdiction de dire que $s$ est inclus dans telle catégorie.

$$
\mathrm{O}\{\mathrm{R}(\mathrm{s}, \sim \operatorname{Resp}) \Rightarrow \mathrm{I}(\ll s \subset C t g »)\}
$$

Par règle disqualificatoire, nous entendons toute règle dont le respect est obligatoirement suffisant pour qu'il soit interdit d'attribuer à un sujet tel qualificatif.

$\mathrm{R}$ est une règle disqualificatoire, lorsque la formule suivante est vérifiée : Il est obligatoire d'interdire de dire que $s$ est inclus dans telle catégorie, s'il a respecté telle(s) règle(s).

$\mathrm{O}\{\mathrm{I}(\ll s \subset C t g ») \operatorname{si} \mathrm{R}(\mathrm{s}, \mathrm{Resp})\} \Leftrightarrow$

$\mathrm{O}\{\mathrm{R}(\mathrm{s}, \mathrm{Resp}) \Rightarrow \mathrm{I}(\ll s \subset C \operatorname{ctg} »)\}$

Remarque: Toute règle disqualificatoire peut être équivalente à une règle qualificatoire opposée (une obligation devient une interdiction, et inversement). C'est alors le non respect de la règle qui devient obligatoire, mais non obligatoirement suffisant pour qu'il soit permis d'attribuer à un sujet tel qualificatif.

$$
\mathrm{O}\{\mathrm{P}(\ll s \subset C t g ») \text { si } \mathrm{R}(\mathrm{s}, \sim \operatorname{Resp})\}
$$

Signalons par ailleurs que le respect ou non des règles qualificatoires n'est pas obligatoirement suffisant pour qu'un sujet ou un objet soit reconnu avec tel qualificatif, encore faut-il que le milieu social ait respecté d'autres règles que nous appelons règles de reconnaissance.

\footnotetext{
${ }^{10}$ Cette notation permet de distinguer ce marqueur de celui de la condition suffisante « Si et seulement si » noté ssi (nous ne parlons pas de condition nécessaire, car c'est souvent l'obligation et non pas la nécessité qui régit la condition, comme c'est le cas ici). Cette distinction n'a pas toujours été effectuée par les travaux en déontique conditionnelle, notamment par ses pionniers (Von Wright, Rescher, Ziemba, Castañeda). Pour une présentation et une discussion de ces travaux, voir Kalinowski, 1972.
} 


\section{Les règles de reconnaissance}

Pour être dit «de qualité $\mathrm{x}$ », il existe des règles pour tous ceux qui pourraient le qualifier comme tel. A l'évidence, n'importe qui ne peut pas identifier untel avec n'importe quel qualificatif, encore faut-il que cette identification soit approuvée. De même d'ailleurs pour les objets, on ne peut pas nommer n'importe quoi avec n'importe quelle étiquette. Il existe donc des règles de reconnaissance qui dépendent, mais restent néanmoins différentes, des règles qualificatoires propres à chaque catégorie.

Or ces règles de reconnaissance ne peuvent jouer ou exister socialement que $s$ 'il est accepté que l'on dise que les règles qualificatoires ont été remplies, ce qui nécessite le plus souvent que des sujets les aient observées ou prouvées.

En supposant qu'une acceptation revient à énoncer une permission (notée $\ll \mathrm{P}(\ldots) »)$, on peut au moins distinguer trois types de règles de reconnaissance.

- Les règles de reconnaissance admises sur de simples ouï-dire :

$\mathrm{R}\left\{(« x \subset C t g »)\right.$ si $\left.\left[S_{i}, \ll \mathrm{P}\left(S_{j}, \ll \mathrm{x}, \mathrm{R} 1 \& \mathrm{R} 2 \& \ldots \& \mathrm{Rn} »\right) »\right]\right\}$

- Les règles de reconnaissance admises à partir d'observations (notées Obs) :

$\mathrm{R}\left\{(\ll x \subset C t g »)\right.$ si $\left.\left\{S_{i}, \ll \mathrm{P}\left[S_{j}, \ll S_{k}, \operatorname{Obs}(\mathrm{x}, \mathrm{R} 1 \& \mathrm{R} 2 \& \ldots \& \mathrm{Rn}) »\right] »\right\}\right\}$

- Les règles de reconnaissance admises à partir de preuves (notées $\operatorname{Pr}$ ) :

$\mathrm{R}\left\{(\ll x \subset C t g »)\right.$ si $\left.\left\{S_{i}, \ll \mathrm{P}\left[S_{j}, \ll S_{k}, \operatorname{Pr}(\mathrm{x}, \mathrm{R} 1 \& \mathrm{R} 2 \& \ldots \& \mathrm{Rn}) »\right] »\right\}\right\}$

On remarquera que l'attribution d'un qualificatif quelconque est donc aussi bien conditionné par l'acceptation, les dires ou le respect de toutes les règles qualificatoires, ou même parfois par leur observation ou leur preuve

Toutes ces règles qui concernent le sujet ou l'objet candidat à l'attribution de telle étiquette impliquent une longue série de règles possibles pour les sujets. Ne pouvant toutes les énumérer, nous prendrons, par exemple, une règle de reconnaissance par observations. Nous avons alors : L'obligation de reconnaissance (= interdiction de dénégation) $\mathrm{O}\{(S, \ll x \subset C \operatorname{tg} ») \ldots$

- s'il a été admis d'avoir dit que le respect de toutes les règles a été observé.

si $\left\{S_{i}, \ll \mathrm{P}\left[S_{j}, \ll S_{k}, \operatorname{Obs}(\mathrm{x}, \mathrm{R} 1 \& \mathrm{R} 2 \& \ldots \& \mathrm{Rn}, \mathrm{Resp})\right.\right.$, Acp », Acp $] »$, Acp $\left.\}\right\}$

- s'il a été admis d'avoir dit que le non respect de l'une des règles n'a pas été observé.

si $\left\{S_{i}, \ll \mathrm{P}\left[S_{j}, \ll S_{k}, \operatorname{Obs}(\mathrm{x}, \mathrm{R} 1 \& \mathrm{R} 2 \& \ldots \& \mathrm{Rn}, \sim \operatorname{Resp}), \sim\right.\right.$ Acp », Acp $] »$, Acp $\left.\}\right\}$

- s'il a été admis de ne pas avoir dit que le non respect de l'une des règles a été observé.

si $\left\{S_{i}, \ll \mathrm{P}\left[S_{j}, \ll S_{k}, \operatorname{Obs}(\mathrm{x}, \mathrm{R} 1 \& \mathrm{R} 2 \& \ldots \& \mathrm{Rn}, \sim \operatorname{Resp})\right.\right.$, Acp $», \sim$ Acp $] »$, Acp $\left.\}\right\}$

- s'il a été admis de ne pas avoir dit que le respect de toutes les règles n'a pas été observé.

si $\left\{S_{i}, \ll \mathrm{P}\left[S_{j}, \ll S_{k}, \operatorname{Obs}(\mathrm{x}, \mathrm{R} 1 \& \mathrm{R} 2 \& \ldots \& \mathrm{Rn}, \operatorname{Resp}), \sim\right.\right.$ Acp $», \sim$ Acp $] »$, Acp $\left.\}\right\}$

- s'il n'a pas été admis d'avoir dit que le respect de toutes les règles n'a pas été observé.

si $\left.\left\{S_{i}, \ll \mathrm{P}\left[S_{j}, \ll S_{k}, \operatorname{Obs}(\mathrm{x}, \mathrm{R} 1 \& \mathrm{R} 2 \& \ldots \& \mathrm{Rn}, \mathrm{Resp}), \sim \mathrm{Acp} », \mathrm{Acp}\right] », \sim \mathrm{Acp}\right\}\right\}$

- s'il n'a pas été admis d'avoir dit que le non respect de l'une des règles a été observé.

si $\left\{S_{i}, \ll \mathrm{P}\left[S_{j}, \ll S_{k}, \operatorname{Obs}(\mathrm{x}, \mathrm{R} 1 \& \mathrm{R} 2 \& \ldots \& \mathrm{Rn}, \sim \operatorname{Resp})\right.\right.$, Acp », Acp $] », \sim$ Acp $\left.\}\right\}$

- s'il a été admis d'avoir dit que le non respect de l'une des règles n'a pas été observé.

si $\left\{S_{i}, \ll \mathrm{P}\left[S_{j}, \ll S_{k}, \operatorname{Obs}(\mathrm{x}, \mathrm{R} 1 \& \mathrm{R} 2 \& \ldots \& \mathrm{Rn}, \sim \operatorname{Resp}), \sim\right.\right.$ Acp $»$, Acp $] »$, Acp $\left.\}\right\}$

- s'il n'a pas été admis de ne pas avoir dit (= s'il a été commandé d'avoir dit) que le respect de toutes les règles a été observé.

si $\left\{S_{i}, \ll \mathrm{P}\left[S_{j}, \ll S_{k}, \operatorname{Obs}(\mathrm{x}, \mathrm{R} 1 \& \mathrm{R} 2 \& \ldots \& \mathrm{Rn}, \operatorname{Resp})\right.\right.$, Acp », $\sim$ Acp $\left.\left.] », \sim \operatorname{Acp}\right\}\right\}$

- s'il n'a pas été admis de ne pas avoir dit que le non respect de l'une des règles n'a pas été observé.

si $\left.\left\{S_{i}, \ll \mathrm{P}\left[S_{j}, \ll S_{k}, \operatorname{Obs}(\mathrm{x}, \mathrm{R} 1 \& \mathrm{R} 2 \& \ldots \& \mathrm{Rn}, \sim \operatorname{Resp}), \sim \mathrm{Acp} », \sim \mathrm{Acp}\right] », \sim \mathrm{Acp}\right\}\right\}$

Nous avons aussi l'interdiction de reconnaissance (= obligation de dénégation)

$\mathrm{I}\{(S, \ll s \subset \mathrm{Ctg} ») \ldots$ 
- s'il n'a pas été admis d'avoir dit que le respect de toutes les règles a été observé

si $\left\{S_{i}, \ll \mathrm{P}\left[S_{j}, \ll S_{k}, \operatorname{Obs}(\mathrm{x}, \mathrm{R} 1 \& \mathrm{R} 2 \& \ldots \& \mathrm{Rn}, \mathrm{Resp})\right.\right.$, Acp », Acp $] », \sim$ Acp $\left.\}\right\}$

- s'il a été admis de ne pas avoir dit (d'avoir caché ou nié) que le non respect de l'une des règles a été observé.

si $\left\{S_{i}, \ll \mathrm{P}\left[S_{j}, \ll S_{k}, \operatorname{Obs}(\mathrm{x}, \mathrm{Rj}, \operatorname{Resp})\right.\right.$, Acp », $\left.\left.\left.\sim \mathrm{Acp}\right] », \operatorname{Acp}\right\}\right\}$

- s'il a été admis d'avoir dit que le non respect de l'une des règles a été observé.

si $\left\{S_{i}, \ll \mathrm{P}\left[S_{j}, \ll S_{k}, \operatorname{Obs}(\mathrm{x}, \mathrm{R} 1 \& \mathrm{R} 2 \& \ldots \& \mathrm{Rn}, \sim \operatorname{Resp})\right.\right.$, Acp », Acp $] », \sim$ Acp $\left.\}\right\}$

- s'il a été admis d'avoir dit que le respect de toutes les règles n'a pas été observé

si $\left\{S_{i}, \ll \mathrm{P}\left[S_{j}, \ll S_{k}, \operatorname{Obs}(\mathrm{x}, \mathrm{R} 1 \& \mathrm{R} 2 \& \ldots \& \mathrm{Rn}, \mathrm{Resp})\right.\right.$, Acp », $\sim$ Acp $] »$, Acp $\left.\}\right\}$

- s'il a été admis d'avoir dit que le respect de toutes les règles n'a pas été observé

si $\left\{S_{i}, \ll \mathrm{P}\left[S_{j}, \ll S_{k}, \operatorname{Obs}(\mathrm{x}, \mathrm{R} 1 \& \mathrm{R} 2 \& \ldots \& \mathrm{Rn}, \mathrm{Resp}), \sim\right.\right.$ Acp , Acp $] »$, Acp $\left.\}\right\}$

- s'il n'a pas été admis d'avoir dit que le non respect de l'une des règles n'a pas été observé.

si $\left\{S_{i}, \ll \mathrm{P}\left[S_{j}, \ll S_{k}, \operatorname{Obs}(\mathrm{x}, \mathrm{R} 1 \& \mathrm{R} 2 \& \ldots \& \mathrm{Rn}, \sim \operatorname{Resp}), \sim \mathrm{Acp} », \mathrm{Acp}\right] », \sim\right.$ Acp $\left.\}\right\}$

- s'il n'a pas été admis de ne pas avoir dit (= s'il a été commandé d'avoir dit) que le non respect de

l'une des règles a été observé.

si $\left\{S_{i}, \ll \mathrm{P}\left[S_{j}, \ll S_{k}, \operatorname{Obs}(\mathrm{x}, \mathrm{R} 1 \& \mathrm{R} 2 \& \ldots \& \mathrm{Rn}, \sim \operatorname{Resp})\right.\right.$, Acp », $\sim$ Acp $] », \sim$ Acp $\left.\}\right\}$

- s'il a été admis de ne pas avoir dit que le non respect de l'une des règles n'a pas été observé.

si $\left\{S_{i}, \ll \mathrm{P}\left[S_{j}, \ll S_{k}, \operatorname{Obs}(\mathrm{x}, \mathrm{R} 1 \& \mathrm{R} 2 \& \ldots \& \mathrm{Rn}, \sim \operatorname{Resp}), \sim\right.\right.$ Acp », $\sim$ Acp $] »$, Acp $\left.\}\right\}$

- s'il n'a pas été admis de ne pas avoir dit que le respect de toutes les règles n'a pas été observé

si $\left\{S_{i}, \ll \mathrm{P}\left[S_{j}, \ll S_{k}, \operatorname{Obs}(\mathrm{x}, \mathrm{R} 1 \& \mathrm{R} 2 \& \ldots \& \mathrm{Rn}, \mathrm{Resp}), \sim \mathrm{Acp} », \sim \mathrm{Acp}\right] », \sim\right.$ Acp $\left.\}\right\}$

Bien entendu, chacun peut aisément retrouver les formules des règles de reconnaissance par ouï-dire ou par preuve qui sont similaires.

On remarquera que l'obligation de reconnaissance (ou l'interdiction de non reconnaissance) est vérifiée si nous trouvons un nombre pair de négations parmi les conditions suivantes :

- L'accomplissement de l'acceptation,

- L'accomplissement de l'énonciation,

- Le respect de toutes les règles,

- L'accomplissement des observations.

L'interdiction de reconnaissance (ou l'obligation de non reconnaissance) est vérifiée si nous trouvons un nombre impair de négations parmi ces mêmes conditions. Ceci est lié aux lois sur les itérations de règles et d'actes (par exemple, il est interdit de permettre de ne pas faire ; il n'est pas obligatoire d'interdire de ne pas faire faire, etc.) : une non négation équivaut à une affirmation, la non négation d'une non négation équivaut aussi une affirmation, etc.

\section{Un exemple : le statut de soigneur}

Pour être soigneur, il faut respecter les règles qualificatoires suivantes :

- L'obligation d'être un homme.

$\mathrm{R} 1=\mathrm{O}(s \subset \mathrm{Ho})$

- L'obligation d'avoir respecté les règles de la catégories des hommes selon la répartition sexuelle des tâches (notamment les règles $\mathrm{R} 1$ à $\mathrm{R} 21$ vues précédemment).

$\mathrm{R} 2=\mathrm{O}\{\mathrm{s}, \mathrm{R} 1(\mathrm{Ho}) \& \mathrm{R} 2(\mathrm{Ho}) \& \ldots \& \mathrm{Rn}(\mathrm{Ho}), \mathrm{Resp}\} \Leftrightarrow$

$=\mathrm{O}\{\mathrm{s}, \quad \Sigma \mathrm{R}(\mathrm{Ho}), \mathrm{Resp}\}$

- L'obligation d'avoir été désigné « soigneur» par un soigneur durant sa petite enfance, lors du baptême traditionnel.

$\mathrm{R} 3=\mathrm{O}\{\mathrm{s}$, [so, Designer «so », Acp $]\}$

Notons bien que le sujet n'est plus l'agent, mais le receveur de l'action ; l'intitulé du verbe (agentif) n'est donc pas placé après lui, mais après le complément, c'est-à-dire ici le soigneur (noté so) situé entre crochets immédiatement après le sujet $\mathrm{s}$. 
- L'obligation d'avoir été préparé chamaniquement par un soigneur pour devenir soigneur. $^{11}$

$\mathrm{R} 4=\mathrm{O}\{\mathrm{s}$, [so, Préparer_so, Acp $]\}$

Avec Préparer_so : la préparation pour devenir soigneur.

- L'obligation d'avoir respecté l'ensemble des règles d'apprentissage des soins d'un soigneur sur des sujets malades.

$\mathrm{R} 5=\mathrm{O}\{\mathrm{s}, \quad \Sigma \mathrm{R}\{$ Apprendre[so, Soigner $(S \subset \mathrm{Ml})]$, Acp $\}, \operatorname{Resp}\}$

Dire que des sujets sont malades équivaut à les inclure dans la catégorie malade (notée Ml) : $S \subset \mathrm{Ml}$

- L'obligation de montrer régulièrement devant témoins (disons des Yucuna, notés $Y u$ ) des soins de sujets malades, suivis de diminutions de la gravité de leur maladie.

$\mathrm{R} 6=\mathrm{O}\left\{\right.$ so, $Y u, \operatorname{Montrer}\left\{\left[\right.\right.$ so, $\left.\left.\left.S, \operatorname{Soigner}(S \subset \mathrm{Ml}), \mathrm{t}_{\mathrm{i}}\right] \&\left[S \subset(\mathrm{Ml}+\backsim \mathrm{Ml}-), \mathrm{t}_{\mathrm{i}+1}\right]\right\}, \operatorname{rglmt}\right\}$

Avec $\mathrm{t}_{\mathrm{i}}$ : temps i (quelconque) $\mathrm{t}_{\mathrm{i}+1}$ : temps $\mathrm{i}+1 ;$ rglmt $: 1$ 'aspect régulier des actes.

La diminution de la gravité d'une maladie est notée par une flèche symbolisant la variation de forme : Ml+ $\leadsto \mathrm{Ml}-$

Avec cette dernière règle qui constitue l'une des règles qualificatoires de l'efficacité thérapeutique, nous avons pris la précaution de ne pas insérer de rapport de cause à effet entre le traitement et l'amélioration de la santé du malade. Nous n'avons ici que l'exhibition d'une relation de concordance faisant parti des règles qualificatoires. En outre, cette règle est doublée d'une obligation de régularité dont la sanction est disqualificatoire, ce qui signifie que l'arrêt des manifestations de cette concordance peut faire perdre au prétendant soigneur sa qualité ou sa renommée.

Mais en prenant une telle précaution, nous ne disons rien sur ce qui fait qu'un soigneur est considéré comme tel. En effet, même une concordance très forte (qui reste encore à remarquer) ne pose jamais à elle-seule l'hypothèse que le traitement du soigneur peut avoir des effets bénéfiques sur le malade.

Cette hypothèse fait alors appel aux règles de reconnaissance de l'efficacité thérapeutique qui fonctionnent par des actes de parole performatifs (Austin, 1970 ; Searle, $1972 ; 1998)$ :

- L'obligation que des Yucuna disent régulièrement que les soins du soigneur peuvent avoir pour effets de diminuer la gravité d'une maladie.

$$
\mathrm{O}\left\{Y u, \ll \text { so, Soigner }\left\{S \subset\left[\left(\mathrm{Ml}+, \mathrm{t}_{\mathrm{i}}\right) \leadsto \diamond\left(\mathrm{Ml}-, \mathrm{t}_{\mathrm{i}+1}\right)\right]\right\} », \operatorname{rglmt}\right\}
$$

Indiquons qu'il est possible d'habiller cette règle de reconnaissance du milieu social en règle qualificatoire pour le sujet. Il suffit simplement de placer le sujet en tant que receveur d'une action indépendante de sa volonté :

- L'obligation pour le soigneur que des Yucuna disent régulièrement que ses soins peuvent avoir pour effets de diminuer la gravité d'une maladie.

$\mathrm{R} 7=\mathrm{O}\left\{\right.$ so, $\left[\mathrm{Yu}, \ll\right.$ so, Soigner $\left.\left.\left\{S \subset\left[\left(\mathrm{Ml}+, \mathrm{t}_{\mathrm{i}}\right) \leadsto \diamond\left(\mathrm{Ml}-, \mathrm{t}_{\mathrm{i}+1}\right)\right]\right\} », \mathrm{rglmt}\right]\right\}$

A partir de la règle de reconnaissance, nous avons ainsi tiré une seconde règle qualificatoire de l'efficacité thérapeutique.

Reste alors à savoir plus précisément ce qui est dit pour expliquer cette hypothèse. Cela nous renvoie à la mythologie ou à des exégèses chamaniques que nous ne pouvons pas reproduire ici. Avant d'approfondir nos recherches en faisant référence à cette tradition orale, nous nous contenterons pour l'instant de traduire et d'intégrer à l'analyse certains concepts clés.

${ }^{11}$ Ce qui, comme dans beaucoup d'autres sociétés d'Amazonie du Nord-Ouest, requière l'initiation au rite de Yurupari. 
Les Yucuna disent qu'un soigneur est apte à «détruire» (kapichátakaje) à l'intérieur du patient l'agent pathogène conceptualisé comme une «flèche » dont l'esprit est un «serpent ».

Le traitement se résume alors à l'équivalence :

$\{$ so, $\mathrm{s}$, Traiter_so $\}=\left\{\right.$ so, $\{$ Détruire, $x[\rightarrow(\subset s)]\}\left\{\left\{s\left[\left(\subset \mathrm{Ml}_{+}, \mathrm{t}_{\mathrm{i}}\right) \leadsto\left(\subset \mathrm{Ml}-, \mathrm{t}_{\mathrm{i}+1}\right)\right]\right\}\right\}$

Avec $x[\rightarrow(\subset s)]$ : agent pathogène $x$ s'étant introduit dans le corps du sujet $s$

$(\subset s)$ : dans le corps du sujet.

$\rightarrow$ : Marqueur de déplacement.

$u$ : Marqueur de variation de forme.

Le soin détruit l'agent pathogène introduit dans le sujet. Ce qui peut faire varier la forme de ce sujet par une diminution de la gravité de sa maladie.

\section{Extrait d'interactions verbales}

Pour montrer de quelle manière ces formules peuvent être utilisées pour analyser des conversations en situations réelles, nous prendrons comme exemple un court extrait d'échanges verbaux recueilli dans un contexte domestique en février $2002 .^{12}$ Ruben fait remarquer à Luz, sa femme, l'importance de la fièvre de leur enfant, en présence du père de celle-ci, Milciades, un soigneur renommé.
1. RUBEN
Le petit a trop de fièvre.
Demasiado to'jmá jelo'kó. ${ }^{13}$
2. LUZ
Oui, il a beaucoup de fièvre.
A’a, kajrú relo'kó.
3. MILCIADES Quel boa lui donne cette fièvre ? Na jewé chi relo'o ? ${ }^{14}$
4.
Le boa de la grippe?
Mu'jrí ta jewé ?

Même un extrait aussi bref peut décourager les tentatives d'analyse par le nombre et la complexité des relations entre les règles supposées ou employées. D'où la nécessité d'une méthode formelle systématique. De plus amples discussions théoriques s'appuyant sur de nombreux exemples recueillis dans des situations variées permettraient d'approfondir et de développer les analyses que nous sommes contraint de limiter ici.

1) Par sa déclaration, Ruben suppose plusieurs types de règles.

- L'obligation pour un père (noté Pe) d'observer (par sensation tactile) tout excès trop important de température (fièvre) fourni par son enfant (noté enf).

$\mathrm{O}\left\{\mathrm{Pe}, \mathrm{Obs}\left(e n f \subset \mathrm{Fv}_{39^{\circ}}\right)\right\}$

avec $\mathrm{Fv}_{39^{\circ}}$ : l'ensemble des sujets très fiévreux (disons d'une température supérieure à $\left.39^{\circ} \mathrm{C}\right)$.

- L'obligation pour un sujet $\mathrm{x}$ (dans ce cas, le père) de reconnaître (= dire qu'il permet de dire) qu'un excès de température chez un sujet y (en l'occurrence l'enfant) peut aboutir soit à un handicap, soit à la mort du sujet.

$\mathrm{O}\left\{\mathrm{x}, \ll \mathrm{P}\left\{\ll\left[y \subset \mathrm{Fv}_{39^{\circ}+}, \mathrm{t}_{0}\right] \leadsto \diamond\left[y \subset\left(\right.\right.\right.\right.$ Handicap W Mort), $\left.\left.\left.\mathrm{t}_{1}\right] »\right\} »\right\}$

$\rightsquigarrow$ : Flèche symbolisant une variation de forme.

\footnotetext{
${ }^{12}$ Ces échanges de parole sont extraits d'une conversation que nous avons filmée. La présentation de sa situation d'énonciation et l'étude des autres échanges englobants participent à l'élaboration d'un ouvrage encore en cours.

${ }^{13}$ Ruben est Tanimuca. Il ne maîtrise pas très bien le Yucuna, et comme beaucoup d'autres jeunes, il place souvent des mots espagnols dans ses phases, surtout s'ils n'ont pas d'équivalent en langue yucuna. C'est le cas avec le terme espagnol demasiado («trop »), généralement utilisé pour signifier que l'excès entraîne des effets. En Yucuna, on ne dit que kajrú («beaucoup ») ou kajrú wani (« très beaucoup »).

${ }^{14}$ Na jewé chi relo'o? Litteralement : Quel boa INTERROGATIF il-a-de-la fièvre. « De quel boa a-t-il de la fièvre ? ».
} 
$\mathrm{t}_{0}$ et $\mathrm{t}_{1}$ : deux moments quelconques se succèdent dans le temps.

Handicap : Ensemble des sujets souffrant d'une incapacité physique

Mort : Ensemble des sujets défunts.

Le symbole $\diamond$ marque la possibilité (et non la nécessité) d'une variation de forme.

- La dévalorisation qu'un sujet perde ses potentialités physiques ou la vie.

$\mathrm{D}\{\mathrm{y},\{\leadsto[\subset$ (Handicap W Mort) $]\}\}$

On peut remarquer que cette dévalorisation est impliquée par l'inaptitude du sujet à réaliser les obligations de la répartition des tâches. Ce qui correspond à une loi logique des rapports de cause à effet entre les règles déontiques et les règles axiologiques :

- Toute cause de l'impossibilité (notée $\sim \diamond$ ) d'accomplir une obligation implique la dévalorisation de cette cause. ${ }^{15}$

$\left\{\left[\mathrm{q}, \mathrm{t}_{0}\right] \Rightarrow \sim \diamond \mathrm{O}\left(\mathrm{t}_{1}, \mathrm{Acp}\right)\right\} \Rightarrow \mathrm{Dq}$

Avec q, un événement quelconque. Pour le considérer en tant que cause, on peut l'inscrire dans le temps en $\mathrm{t}_{0}$ avec son effet en $\mathrm{t}_{1}$

Nous avons donc :

$\{\mathrm{y},\{\leadsto[\subset$ (Handicap W Mort) $\} \Rightarrow \sim \diamond \Sigma \mathrm{O}(\mathrm{Y})\}\} \Rightarrow \mathrm{D}\{\mathrm{y},\{\leadsto[\subset$ (Handicap W Mort) $]\}\}$

Mais sans doute pourrait-on définir d'autres règles impliquant elles aussi cette dévalorisation.

En raison des règles qualificatoires d'un soigneur, nous avons également :

- L'obligation pour un soigneur de soigner un sujet dont on a déclaré l'excès de fièvre.

$\mathrm{O}\left\{\left[\right.\right.$ so, $\left.\operatorname{Soigner}\left(S \subset \mathrm{Fv}_{39^{\circ}+}\right)\right]$ si $\left.\left[\mathrm{x}, \ll S \subset \mathrm{Fv}_{39^{\circ}+}{ }\right]\right\}$

Comme l'ensemble des sujets trop fiévreux est inclus dans l'ensemble des sujets malades (Soit : $\mathrm{Fv}_{39^{\circ}+} \subset \mathrm{Ml}$ ), cette obligation est impliquée par la règle R6 du soigneur :

- L'obligation pour un soigneur de montrer devant témoins qu'il soigne régulièrement des sujets malades.

$\mathrm{R} 6=\mathrm{O}\left\{\right.$ so, $Y u, \operatorname{Montrer}\left\{\left[\right.\right.$ so, $\left.\left.\left.S, \operatorname{Soigner}(S \subset \mathrm{Ml}), \mathrm{t}_{\mathrm{i}}\right] \&\left[S \subset(\mathrm{Ml}+\leadsto \mathrm{Ml}-), \mathrm{t}_{\mathrm{i}+1}\right]\right\}, \mathrm{rglmt}\right\}$

$\mathrm{R} 6 \Rightarrow \mathrm{O}\{s o, Y u$, Montrer[so, Soigner $(S \subset \mathrm{Ml})]$, rglmt $\}$

$\mathrm{R} 6 \Rightarrow \mathrm{O}\left\{s o, Y u\right.$, Montrer[so, Soigner $\left.\left(S \subset \mathrm{Fv}_{39^{\circ}+}\right)\right]$, rglmt $\}$

$\mathrm{R} 6 \Rightarrow \mathrm{O}\left\{\right.$ so, Soigner $\left.\left(S \subset \mathrm{Fv}_{39^{\circ}}\right)\right]$, rglmt $\}$

$\mathrm{R} 6 \Rightarrow \mathrm{O}\left\{\left[\right.\right.$ so, $\left.\operatorname{Soigner}\left(S \subset \mathrm{Fv}_{39^{\circ}+}\right)\right]$ si $\left.\left[\mathrm{x}, \ll S \subset \mathrm{Fv}_{39^{\circ}+}{ }\right]\right\}$

Or si Ruben sait qu'une telle déclaration entraîne une telle obligation ayant pour effet d'éviter un événement dramatique, on peut penser qu'il la réalise avec cette finalité. Il valorise un acte empêchant une dévalorisation. Cela correspond à une loi logique des rapports de cause à effet entre les règles axiologiques :

Toute cause de l'impossibilité d'accomplir une dévalorisation implique la valorisation de

${ }^{15}$ Notons d'autres lois de même type :

- Toute cause de la nécessité d'accomplir une obligation implique la valorisation de cette cause.

$\left\{\left[\mathrm{q}, \mathrm{t}_{0}\right] \Rightarrow \mathrm{O}\left(\mathrm{t}_{1}, \mathrm{Acp}\right)\right\} \Rightarrow \mathrm{Vq}$

- Toute cause de l'impossibité d'accomplir un interdit implique la valorisation de cette cause.

$\left\{\left[\mathrm{q}, \mathrm{t}_{0}\right] \Rightarrow \sim \mathrm{I}\left(\mathrm{t}_{1}\right.\right.$, Acp $\left.)\right\} \Rightarrow \mathrm{Vq}$

- Toute cause de la nécessité d'accomplir une interdiction implique la dévalorisation de cette cause.

$\left\{\left[\mathrm{q}, \mathrm{t}_{0}\right] \Rightarrow \mathrm{I}\left(\mathrm{t}_{1}, \mathrm{Acp}\right)\right\} \Rightarrow \mathrm{Dq}$ 
cette cause. $^{16}$

$\left\{\left[\mathrm{q}, \mathrm{t}_{0}\right] \Rightarrow \sim \diamond \mathrm{D}\left(\mathrm{t}_{1}, \mathrm{Acp}\right)\right\} \Rightarrow \mathrm{Vq}$

La déclaration de Ruben est donc réalisée par valorisation, en raison de l'obligation qu'elle pose :

$\left\{\mathrm{x}, \ll S \subset \mathrm{Fv}_{39^{\circ}+}, \mathrm{t}_{0} », \mathrm{t}_{1}\right\} \Rightarrow \mathrm{O}\left\{\left[\right.\right.$ so, Soigner $\left.\left.\left(S \subset \mathrm{Fv}_{39^{\circ}+}\right)\right], \mathrm{t}_{2}\right\} \Rightarrow \sim \diamond \mathrm{D}\left[S \subset\right.$ (Handicap W Mort), $\left.\mathrm{t}_{3}\right]$

$\Rightarrow \mathrm{V}\left\{\mathrm{x}, \ll S \subset \mathrm{Fv}_{39^{\circ}+}, \mathrm{t}_{0} », \mathrm{t}_{1}\right\}$

Ruben (noté $r u$ ) réalise ainsi la valorisation :

$\mathrm{V}\left\{r u\right.$, «enf $\left.\subset \mathrm{Fv}_{39^{\circ}+}, \mathrm{t}_{0} », \mathrm{Acp}\right\}$

2) Luz confirme l'énoncé précédent, ce qui suppose d'une part:

- La dévalorisation qu'un sujet $\mathrm{x}$ déclare la fièvre du sujet $\mathrm{y}$ au moment $\mathrm{t}_{0}$ alors que d'autres témoins $Z$ reconnaissent (= admettent de dire) qu'ils n'ont pas observé d'excès de fièvre à ce moment.

$\mathrm{D}\left\{\mathrm{x}, \ll y \subset \mathrm{Fv}_{39^{\circ}+}, \mathrm{t}_{0} », \operatorname{si}\left\{Z, \ll \mathrm{P}\left[\ll Z, \sim \operatorname{Obs}\left(y \subset \mathrm{Fv}_{39^{\circ}+}, \mathrm{t}_{0}\right) »\right] »\right\}\right\}$

Cette dévalorisation est impliquée par un interdit de reconnaissance par observation :

- Il est interdit (donc dévalorisé) de dire qu'un sujet est inclu dans telle catégorie, s'il est reconnu que les règles qualificatoires n'ont pas été observées.

$\mathrm{I}\left\{\mathrm{x}, \ll y \subset \mathrm{Fv}_{39^{\circ}+}, \mathrm{t}_{0} »\right.$, si $\left.\left\{Z, \ll \mathrm{P}\left[\ll Z, \sim \operatorname{Obs}\left(y \subset \mathrm{Fv}_{39^{\circ}+}, \mathrm{t}_{0}\right) »\right] »\right\}\right\} \Rightarrow$

$\mathrm{D}\left\{\mathrm{x}, \ll y \subset \mathrm{Fv}_{39^{\circ}+}, \mathrm{t}_{0} »\right.$, si $\left.\left\{Z, \ll \mathrm{P}\left[\ll Z, \sim \operatorname{Obs}\left(y \subset \mathrm{Fv}_{39^{\circ}+}, \mathrm{t}_{0}\right) »\right] »\right\}\right\}$

D'autre part, Luz suppose une valorisation :

- La valorisation pour un sujet $\mathrm{z}$ de reconnaître que l'excès de fièvre a été observé ce moment.

$\mathrm{V}\left\{\mathrm{x}, \ll y \subset \mathrm{Fv}_{39^{\circ}+}, \mathrm{t}_{0} », \operatorname{si}\left\{z, \ll \mathrm{P}\left[\ll z, \operatorname{Obs}\left(y \subset \mathrm{Fv}_{39^{\circ}+}, \mathrm{t}_{0}\right) »\right] »\right\}\right\}$

De la même manière, cette valorisation est impliquée par une obligation de reconnaissance par observation :

- Il est obligatoire (donc valorisé) de dire qu'un sujet est inclu dans telle catégorie, s'il est reconnu que les règles qualificatoires ont été observées.

$\mathrm{O}\left\{\mathrm{x}, \ll y \subset \mathrm{Fv}_{39^{\circ}+}, \mathrm{t}_{0} »\right.$, si $\left.\left\{Z, \ll \mathrm{P}\left[\ll Z, \operatorname{Obs}\left(y \subset \mathrm{Fv}_{39^{\circ}+}, \mathrm{t}_{0}\right) »\right] »\right\}\right\} \Rightarrow$

$\mathrm{V}\left\{\mathrm{x}, \ll y \subset \mathrm{Fv}_{39^{\circ}+}, \mathrm{t}_{0} », \operatorname{si}\left\{Z, \ll \mathrm{P}\left[\ll Z, \operatorname{Obs}\left(y \subset \mathrm{Fv}_{39^{\circ}+}, \mathrm{t}_{0}\right) »\right] »\right\}\right\}$

La déclaration de Luz (notée $l u$ ) peut donc être comprise comme l'accomplissement d'une valorisation : $\mathrm{V}\left\{\left\{\mathrm{lu}, \ll e n f \subset \mathrm{Fv}_{39^{\circ}+}, \mathrm{t}_{0} », \operatorname{Acp}\right\}\right.$, si $\left.\left\{l u, \ll \mathrm{P}\left[\ll r u, \operatorname{Obs}\left(e n f \subset \mathrm{Fv}_{39^{\circ}+}, \mathrm{t}_{0}\right) »\right] »\right\}\right\}$

3) Milciades, en tant que soigneur, est obligé d'interroger en raison de la règle R6, vue précédemment (l'effet de la déclaration de Ruben a été d'activer cette règle).

En outre, la règle qualificatoire R5 d'apprentissage que Milciades (noté mi) a reçu, en particulier pour soigner la grippe (notée Ml-g), l'oblige à le faire, si l'occasion se présente.

\footnotetext{
16 En français, on le sous-entend en disant : «C'est pour la bonne cause ».

Notons les autres lois similaires :

- Toute cause de la nécessité d'accomplir une valorisation implique la valorisation de cette cause.

$\left\{\left[\mathrm{q}, \mathrm{t}_{0}\right] \Rightarrow \mathrm{V}\left(\mathrm{t}_{1}, \mathrm{Acp}\right)\right\} \Rightarrow \mathrm{Vq}$

- Toute cause de la nécessité d'accomplir une dévalorisation implique la dévalorisation de cette cause.

$\left\{\left[\mathrm{q}, \mathrm{t}_{0}\right] \Rightarrow \mathrm{D}\left(\mathrm{t}_{1}, \mathrm{Acp}\right)\right\} \Rightarrow \mathrm{Dq}$

- Toute cause de l'impossibilité d'accomplir une valorisation implique la dévalorisation de cette cause.

$\left\{\left[\mathrm{q}, \mathrm{t}_{0}\right] \Rightarrow \sim \mathrm{V}\left(\mathrm{t}_{1}, \mathrm{Acp}\right)\right\} \Rightarrow \mathrm{Dq}$
} 

g\}

Milciades est donc obliger d'interroger précisément Luz et Ruben (notés $l u$ \& $r u$ ) sur la condition qui le concerne en tant que soigneur : «Est-ce que la maladie liée à la fièvre de l'enfant peut être soignée grâce aux compétences qu'il a apprises ? », ce qui revient à questionner plus brièvement : « De quelle maladie souffre l'enfant? ». Soit :

$\mathrm{O}\{m i, l u \& r u, \ll(s \subset \mathrm{Ml}) ? », \mathrm{Acp}\}$

Avec ? : le marqueur d'interrogation sur ce qui précède.

4) Selon sa conception thérapeutique, le boa symbolise pour lui la cause de la fièvre de l'enfant, lorsqu'il demande Na jewé chi relo'o ? "Quel boa lui donne cette fièvre ? », et lorsqu'il demande confirmation de l'hypothèse qu'il s'agit du «boa de la grippe ».

Si c'est ce type de «boa » qui est vu comme le facteur de la maladie, il est obligé de tenter de le soigner. Ce qui l'incite à valoriser l'interrogation sur ce genre d'hypothèse.

Il réalise donc :

$\mathrm{V}\{m i, l u \& r u, \ll(s \subset \mathrm{Ml}-\mathrm{g}) ? », \mathrm{Acp}\}$

****

Dans cet article, nous sommes restés bien loin de pouvoir exposer l'ensemble des possibilités heuristiques et fonctionnelles que les logiques modales offrent aux sciences sociales, et à l'anthropologie en particulier. Toutefois, notre propos n'était ni d'en faire une présentation ou une introduction générale, ni de la développer pour construire un langage idéal. Il s'agissait essentiellement de montrer son intérêt méthodologique pour la systématisation et la comparaison de analyses sur les règles sociales.

Cela s'est fait, nous l'avons vu, au prix d'un formalisme, qui pour certains apparaîtra obscure, réducteur, voire inutile (puisqu'il peut également être traduit dans un langage courant). Néanmoins, nous considérons assez difficile (et même risqué) de s'engager davantage dans ces trois types de critiques.

Tout d'abord, parce que les sciences se sont généralement construites au moyen d'un certain langage formel, qu'elles sont censées adapter à leurs objectifs heuristiques. A l'évidence, elles s'appuient aussi dessus (d'où «l'inutilité heuristique » de certains formalismes) pour faire autorité sur d'autres formes d'expression relativement plus accessibles chez de larges populations.

Ensuite, parce que l'accusation de «réductionnisme» empêche rarement une théorie ou un paradigme d'éclore et de se développer. Bien au contraire, selon les préceptes de Descartes, les méthodes scientifiques analysent en priorité les «choses simples», au risque de laisser le temps de leurs développements une vision trop «simpliste» de ce qu'elles représentent. C'est même grâce à de telles accusations que les théories sont révisées et complétées, quitte à devenir de plus en plus complexes. Peu importe donc qu'une théorie soit réductionniste (elle l'est nécessairement), l'important c'est sa capacité à analyser relativement simplement une grande quantité de faits.

Enfin, un langage formel n'est pas inutile s'il permet à une science d'exprimer et d'expliquer avec un minimum de moyens linguistiques (signes, notations symboliques) un grand nombre de phénomènes avec davantage de portée et de précision qu'un autre langage.

Néanmoins, on pourra certainement exprimer quelques regrets de ne pas avoir pu pousser plus loin les analyses. En effet, il aurait été intéressant de décrire le système social plus globalement (par exemple, en considérant les institutions occidentales en interactions avec les institutions indigènes) ou de manière plus détaillée, notamment en présentant la répartition des tâches avec les autres statuts que celui des soigneurs (maîtres de maloca, 
chanteur-danseur), les règles de parenté, ou encore les règles particulières à chaque contexte (vie domestique, visites, travaux collectifs, cérémonies).

Par ailleurs, à la différence des échanges de parole brièvement examinés ici, ceux-ci deviennent de plus en plus intéressants à mesure que les extraits commentés s'étendent, et que les analyses se multiplient ou s'enchaînent, tout en mettant en relief leur dynamique séquentielle (Fontaine, 2001). Sans avoir pu exposer davantage les différentes pistes de recherche qu'ouvrent l'étude des règles et de leurs effets sur les actes, nous aurons simplement donné un aperçu assez représentatif des nouvelles possibilités de traitement qu'offre un langage formel basé sur les logiques modales.

\section{Bibliographie}

AUSTIN John Langshaw

1970 Quand dire, c'est faire. Paris, Le Seuil, (Essais).

BAILHACHE Patrice

1991 Essai de logique déontique. Paris, Vrin.

ECO Umberto

1984 La structure absente, Mercure de France.

FONTAINE Laurent

2001 Paroles d'échange et règles sociales chez les Indiens yucuna d'Amazonie colombienne. Thèse Iheal-Paris III.

2002 «La monnaie, une modalité d'échange parmi d'autres chez les Indiens yucuna d'Amazonie colombienne », Journal des anthropologues, $\mathrm{n}^{\circ}$ 90-91 : 171-188.

JACOPIN Pierre-Yves

1981 La parole générative de la mythologie des Indiens Yukuna. Thèse Université de Neuchâtel.

1988 « On the Syntactic Structure of Myth, or the Yukuna Invention of Speech », Cultural Anthropology 3 (2): 131-158

1993-1994 (sous la dir. de) La parole mythique en Amérique du sud, Société Suisse des Américanistes, bulletin 57-58.

LEVI-STRAUSS Claude

1958 Anthropologie structurale, Paris. Plon, (Rééd., 1974).

KALINOWSKI Georges

1972 La logique des normes. Paris, Puf.

1996 La logique déductive. Paris, Puf.

RADCLIFFE-BROWN A. R. 
1968 Structure et fonction dans la société primitive, Paris, Edition de Minuit.

SEARLE John R.

1972 Les actes de langage. Essai de philosophie du langage. Paris, Hermann.

1998 La construction de la réalité sociale. Paris, Gallimard.

WRIGHT Georg Henrik Von

1951 « Deontic logic ». Mind, $60: 1-15$.

\section{Résumé}

Cet article étudie les règles sociales, en exposant par quels moyens formels il est aujourd'hui possible de perfectionner et de systématiser leurs analyses. Ces moyens, nous les devons en particulier à la logique déontique de G. H. Von Wright, qui depuis son célèbre texte de 1951, a connu d'amples développements, notamment en France, grâce à des auteurs comme Kalinowski ou Bailhache. De tels travaux ne concernent pas uniquement les sciences juridiques, mais l'anthropologie dans son ensemble. En prenant comme exemple de société celle des Indiens yucuna de l'Amazonie colombienne, on peut montrer de quelle manière les logiques modales s'avèrent pertinentes non seulement pour identifier les règles qui régissent la répartition des tâches, mais aussi pour étudier leurs effets dans les échanges de paroles. 